\title{
Homogenization of contact problem with Coulomb's friction on periodic cracks
}

\author{
G. Griso, J. Orlik
}

\begin{abstract}
We consider the elasticity problem in a domain with contact on multiple periodic open cracks. The contact is described by the Signorini and Coulomb-friction conditions. Problem is non-linear, the dissipative functional depends on the un-known solution and the existence of the solution for fixed period of the structure is usually proven by the fix-point argument in the Sobolev spaces with a little higher regularity, $H^{1+\alpha}$. We rescaled norms, trace, jump and Korn inequalities in fractional Sobolev spaces with positive and negative exponent, using the unfolding technique, introduced by Griso, Cioranescu and Damlamian. Then we proved the existence and uniqieness of the solution for friction and period fixed. Then we proved the continuous dependency of the solution to the problem with Coulomb's friction on the given friction and then estimated the solution using fixed point theorem. However, we were not able to pass to the strong limit in the frictional dissipative term. For this reason, we regularized the problem by adding a fourth-order term, which increased the regularity of the solution and allowed the passing to the limit. This can be interpreted as micro-polar elasticity.
\end{abstract}

\section{Keywords:}

\section{Mathematics Subject Classification:}

\section{Introduction}

This paper deals with a static multi-scale contact problem with Coulomb's friction, which arise by timediscretization of a quasi-static problem, as it is shown in [3], 4. Such a problem results in a quasi-variational inequality, whose solvability was studied in [3], 4]. In this paper, we want to repeat these results for multi-scale periodic open cracks.

We show that the Coulomb-friction problem admits solutions for every fixed period. Then we obtain all compactness results and preliminary estimates in terms of the powers of the small parameter, related to the period of the structure, using Korn's inequalities and their rescaling. For this reason, we extend results of [10] and [8], where asymptotic analysis and one-side Korn inequalities were given for multi-scale contact problem in a periodic domain with Tresca friction, and extend the unfolding tools introduced by [5] and [7] to fractional order Sobolev spaces. We introduce tools for rescaling their norms and dual norms over domains and manifolds. We regularize the problem by adding the fourth-order term, in order to pass to the limit and prove the strong convergence of the interface stresses or co-normal derivatives on the cracks. We used the shifting technique as in [4], or [15] to show a better regularity in the macroscopic variable and, then, the strong convergence. Finally, we proved the existence and uniqueness of the solution to the limiting regularized problem. It is easy to show that the solution of the regularized $\varepsilon$-problem converges to the solution of the contact Coulomb's problem as the regularizing parameter tends to zero. However, it cannot be sent to zero after the passing to the limit, since it enters a denominator of the contruction condition for the fixed point argument. In the conclusion, we were able to homogenize just a regularized problem. The result is still new and interesting and can be applied to micro-polar materials with some additional rotational degrees of freedom. 


\section{Geometric set up}

The problem is set in the natural space $\mathbb{R}^{3}$. Denote $\Omega$ a bounded domain in $\mathbb{R}^{3}$ with Lipschitz boundary and $\Gamma$ a subset of $\partial \Omega$ with a positive Lebesgue measure on $\partial \Omega$.

In the following $\mathbf{Y} \doteq(0,1)^{3}$, is the reference cell. The crack, is a closed set denoted $S$ and strictly included in $\mathbf{Y}$ and called "open crack". The crack $S$, is "open" in the sense that $\mathbf{Y}^{*}=\mathbf{Y} \backslash S$ lies on both sides of this surface. The set matrix is $\mathbf{Y}^{*} \doteq \mathbf{Y} \backslash S$ (see Figure 1).

We assume that $S$ is the closure of an open connected set of the boundary of a domain $\mathcal{S}$ strictly included in $\mathbf{Y}$ and whose boundary is $\mathcal{C}^{1,1}$. We denote $\nu$ the outward unit normal vector to the boundary of the domain $\mathcal{S}$ (it belongs to $W^{1, \infty}(\partial \mathcal{S})^{3}$ ).

Recall that in the periodic setting, almost every point $z \in \mathbb{R}^{3}$ can be written as

$$
z=[z]+\{z\}, \quad[z] \in \mathbb{Z}^{3}, \quad\{z\} \in \mathbf{Y} .
$$

Denote

$$
\Xi_{\varepsilon}=\left\{\xi \in \mathbb{Z}^{3} \mid \varepsilon \xi+\varepsilon \mathbf{Y} \subset \Omega\right\}, \quad \widehat{\Omega}_{\varepsilon}=\text { interior }\left\{\bigcup_{\xi \in \Xi_{\varepsilon}}(\varepsilon \xi+\varepsilon \overline{\mathbf{Y}})\right\}, \quad \Lambda_{\varepsilon}=\Omega \backslash \widehat{\widehat{\Omega}}_{\varepsilon},
$$

this last set contains the parts from cells intersecting the boundary $\partial \Omega$.

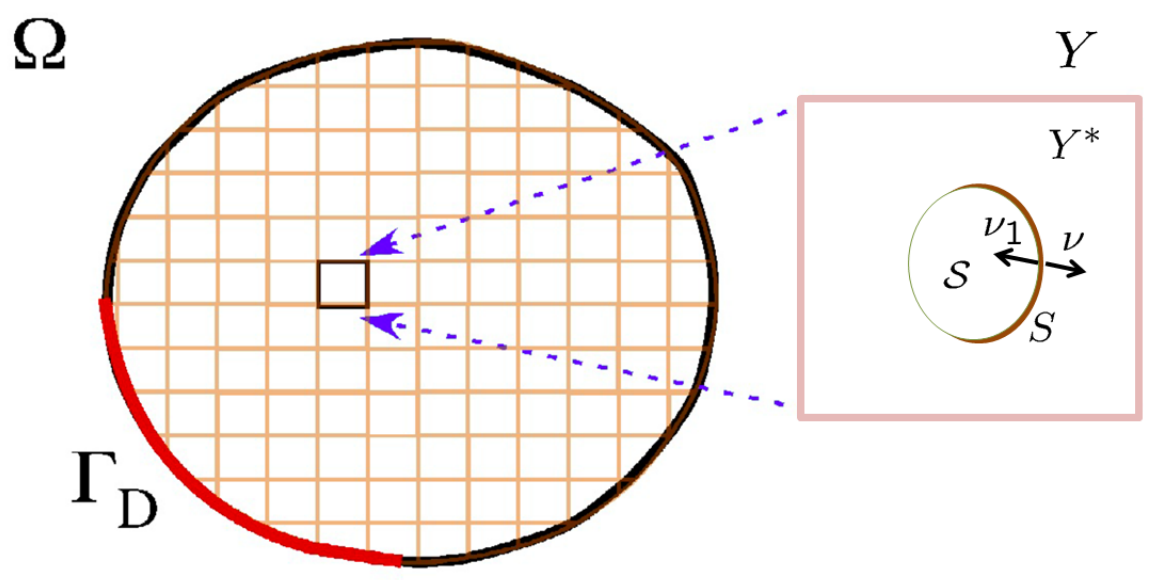

Figure 1. Bounded domain with periodically distributed open and closed cracks

The union of all the cracks is denoted $S_{\varepsilon}$,

$$
S_{\varepsilon} \doteq\left\{x \in \widehat{\Omega}_{\varepsilon} \mid\left\{\frac{x}{\varepsilon}\right\} \in S\right\} \subset \mathcal{S}_{\varepsilon} \doteq\left\{x \in \widehat{\Omega}_{\varepsilon} \mid\left\{\frac{x}{\varepsilon}\right\} \in \mathcal{S}\right\}
$$

and the cracked domain

$$
\Omega_{\varepsilon}^{*}=\Omega \backslash S_{\varepsilon} .
$$

Set

$$
H_{\Gamma}^{1}\left(\Omega_{\varepsilon}^{*}\right)=\left\{u \in H^{1}\left(\Omega_{\varepsilon}^{*}\right) \mid u=0 \text { a.e. on } \Gamma\right\}, \quad H_{\Gamma}^{2}\left(\Omega_{\varepsilon}^{*}\right)=H_{\Gamma}^{1}\left(\Omega_{\varepsilon}^{*}\right) \cap H^{2}\left(\Omega_{\varepsilon}^{*}\right)
$$

\section{Notations}

- The normal component of a vector field $v$ on the boundary of a domain with Lipschitz boundary is denoted $v_{\nu}$, while the tangential component $v-v_{\nu} \nu$ is denoted $v_{\tau_{\varepsilon}}$ (where $\nu$ is the outward unit normal to the boundary),

- the strain tensor of a vector field $v$ is denoted by $e(v)$; its values are symmetric $3 \times 3$ real matrices,

- we use the notations of [5, 7] for the unfolding method. 


\section{Preliminary results}

\subsection{Recall on Poincaré, Poincaré-Wirtinger and Korn inequalities}

In the following, for every open bounded set $\mathcal{O} \subset \mathbb{R}^{3}$ and $\varphi \in L^{1}(\mathcal{O}), \mathcal{M}_{\mathcal{O}}(\varphi)$ denotes the mean value of $\varphi$ over $\mathcal{O}$, i.e.,

$$
\mathcal{M}_{\mathcal{O}}(\varphi)=\frac{1}{|\mathcal{O}|} \int_{\mathcal{O}} \varphi d y
$$

Let $\mathcal{O}$ be a bounded domain in $\mathbb{R}^{3}$ with Lipschitz boundary. In $\mathcal{O}$, the following Poincaré and PoincaréWirtinger inequalities hold:

$$
\begin{array}{ll}
\forall \phi \in H^{1}(\mathcal{O}), \quad & \|\phi\|_{H^{1}(\mathcal{O})} \leq C\left(\|\nabla \phi\|_{L^{2}(\mathcal{O})}+\|\phi\|_{L^{2}(\partial \mathcal{O})}\right), \\
& \left\|\phi-\mathcal{M}_{\mathcal{O}}(\phi)\right\|_{L^{2}(\mathcal{O})} \leq C\|\nabla \phi\|_{L^{2}(\mathcal{O})} .
\end{array}
$$

where the constant $C$ depends on $\mathcal{O}$.

Recall that the space of rigid displacements is

$$
\mathcal{R}=\left\{r \mid r(x)=a+b \wedge x, \quad(a, b) \in \mathbb{R}^{3} \times \mathbb{R}^{3}\right\} .
$$

A bounded domain $\mathcal{O} \subset \mathbb{R}^{3}$ satisfies the Korn-Wirtinger inequality if there exists a constant $C$ such that for every $v \in H^{1}(\mathcal{O})^{3}$ there exists $r \in \mathcal{R}$ such that

$$
\|v-r\|_{H^{1}(\mathcal{O})} \leq C\|e(v)\|_{L^{2}(\mathcal{O})} .
$$

We equip $H^{1}(\mathcal{O})^{3}$ with the scalar product

$$
<<u, v>>=\int_{\mathcal{O}} e(u): e(v) d x+\int_{\mathcal{O}} u \cdot v d x
$$

If $\mathcal{O} \subset \mathbb{R}^{3}$ is a bounded domain with Lipschitz boundary, $\mathcal{O}$ satisfies the Korn-Wirtinger inequality and the associated norm is equivalent to the usual norm of $H^{1}(\mathcal{O})^{3}$. For the displacements in $H^{1}(\mathcal{O})^{3}$ one also has the following Poincaré inequality:

$$
\forall v \in H^{1}(\mathcal{O})^{3}, \quad\|v\|_{H^{1 / 2}(\partial \mathcal{O})} \leq C\left(\|e(v)\|_{L^{2}(\mathcal{O})}+\|v\|_{L^{2}(\mathcal{O})}\right)
$$

where $C$ depends on $\mathcal{O}$.

Definition 1. Let $\mathcal{O}$ be a bounded domain in $\mathbb{R}^{3}$ with Lipschitz boundary, we denote

$$
W(\mathcal{O}) \doteq\left\{v \in H^{1}(\mathcal{O})^{3} \mid \int_{\mathcal{O}} v(x) \cdot r(x) d x=0 \text { for all } r \in \mathcal{R}\right\} .
$$

The space $W(\mathcal{O})$ is the orthogonal of $\mathcal{R}$ in $H^{1}(\mathcal{O})^{3}$ for the scalar product 3.2 .

Recall that there exists a constant $C$ such that for every $v \in W(\mathcal{O})$

$$
\|v\|_{H^{1}(\mathcal{O})} \leq C\|e(v)\|_{L^{2}(\mathcal{O})} .
$$

\subsection{The spaces $H^{\alpha}(S)$ and $H^{\alpha}\left(S_{\varepsilon}\right), \alpha \in(0,1)$}

The space $H^{\alpha}(S), \alpha \in(0,1)$, is the following subspace of $L^{2}(S)$ :

$$
H^{\alpha}(S) \doteq\left\{v \in L^{2}(S) \mid \int_{S} \int_{S} \frac{|v(x)-v(y)|^{2}}{|x-y|^{2+2 \alpha}} d \sigma_{x} d \sigma_{y}<+\infty\right\} .
$$

It is equipped with the semi-norm

$$
\|v\|_{H^{\alpha}(S)}^{2}=\int_{S} \int_{S} \frac{|v(x)-v(y)|^{2}}{|x-y|^{2+2 \alpha}} d \sigma_{x} d \sigma_{y}
$$


and the Sobolev-Slobodetsky norm

$$
\|v\|_{H^{\alpha}(S)}=\sqrt{\|v\|_{L^{2}(S)}^{2}+\|v\|_{H^{\alpha}(S)}^{2}} .
$$

The space $H^{\alpha}\left(S_{\varepsilon}\right), \alpha \in(0,1)$ is the subspace of $L^{2}\left(S_{\varepsilon}\right)$ containing the functions whose restrictions to every connected component of $S_{\varepsilon}$ belong to the space $H^{\alpha}$ of this connected component

$$
H^{\alpha}\left(S_{\varepsilon}\right) \doteq\left\{v \in L^{2}\left(S_{\varepsilon}\right) \mid \sum_{\xi \in \Xi_{\varepsilon}} \int_{\varepsilon \xi+\varepsilon S} \int_{\varepsilon \xi+\varepsilon S} \frac{|v(x)-v(y)|^{2}}{|x-y|^{2+2 \alpha}} d \sigma_{x} d \sigma_{y}<+\infty\right\} .
$$

It is equipped with the semi-norm

$$
\|v\|_{H^{\alpha}\left(S_{\varepsilon}\right)}^{\prime 2}=\sum_{\xi \in \Xi_{\varepsilon}} \int_{\varepsilon \xi+\varepsilon S} \int_{\varepsilon \xi+\varepsilon S} \frac{|v(x)-v(y)|^{2}}{|x-y|^{2+2 \alpha}} d \sigma_{x} d \sigma_{y}
$$

and the Sobolev-Slobodetsky norm

$$
\|v\|_{H^{\alpha}\left(S_{\varepsilon}\right)}=\sqrt{\|v\|_{L^{2}\left(S_{\varepsilon}\right)}^{2}+\varepsilon^{2 \alpha}\|v\|_{H^{\alpha}\left(S_{\varepsilon}\right)}^{\prime 2}} .
$$

\subsection{Definition of the jumps}

For every $v \in H^{1}\left(\mathbf{Y}^{*}\right)$ denote $v_{\partial \mathcal{S}^{-}}$the trace of $v_{\mid \mathcal{S}}$ on $\partial \mathcal{S}$ and $v_{\partial \mathcal{S}^{+}}$the trace of $v_{\mid \mathbf{Y}^{*} \backslash \overline{\mathcal{S}}}$ on $\partial \mathcal{S}$. The jump of $v$ across the surface $S$ is

$$
[v]_{S}=\left(v_{\mid \partial \mathcal{S}^{+}}-v_{\mid \partial \mathcal{S}^{-}}\right)_{\mid S}, \quad[v]_{S} \in \widetilde{H}^{1 / 2}(S)^{1}
$$

We also set for every $v \in H^{1}\left(\mathbf{Y}^{*}\right)^{3}$

$$
\left[v_{\nu}\right]_{S}=[v]_{S} \cdot \nu \in \widetilde{H}^{1 / 2}(S), \quad\left[v_{\tau}\right]_{S}=[v]_{S}-\left[v_{\nu}\right]_{S} \nu \in \widetilde{H}^{1 / 2}(S)^{3} .
$$

In a similar way, for every $v \in H^{1}\left(\Omega_{\varepsilon}^{*}\right)^{3}$ one defines $[v]_{S_{\varepsilon}}$ and $\left[v_{\nu_{\varepsilon}}\right]_{S_{\varepsilon}}$ and $\left[v_{\tau_{\varepsilon}}\right]_{S_{\varepsilon}}$.

\subsection{Some recalls on the main periodic unfolding operators}

We follow the notations and definitions of [7].

Proposition 3.1. Assume $p \in[1,+\infty]$, then

- for every $\phi \in L^{p}\left(\Omega_{\varepsilon}^{*}\right)$ one has (recall that $|\mathbf{Y}|=1$ )

$$
\left\|\mathcal{T}_{\varepsilon}^{*}(\phi)\right\|_{L^{p}\left(\Omega \times \mathbf{Y}^{*}\right)}=\|\phi\|_{L^{p}\left(\widehat{\Omega}_{\varepsilon}^{*}\right)} \leq\|\phi\|_{L^{p}\left(\Omega_{\varepsilon}^{*}\right)} .
$$

- for every $\phi \in W^{1, p}\left(\Omega_{\varepsilon}^{*}\right)$ one has $\nabla_{y} \mathcal{T}_{\varepsilon}^{*}(\phi)(x, y)=\varepsilon \mathcal{T}_{\varepsilon}^{*}(\nabla \phi)(x, y)$ for a.e. $(x, y) \in \Omega \times \mathbf{Y}^{*}$ and

$$
\left\|\nabla_{y} \mathcal{T}_{\varepsilon}^{*}(\phi)\right\|_{L^{p}\left(\Omega \times \mathbf{Y}^{*}\right)}=\varepsilon\|\nabla \phi\|_{L^{p}\left(\widehat{\Omega}_{\varepsilon}^{*}\right)} \leq \varepsilon\|\nabla \phi\|_{L^{p}\left(\Omega_{\varepsilon}^{*}\right)} .
$$

Definition 3.2. Assume $p \in[1,+\infty]$. The operator $\mathcal{T}_{\varepsilon}^{b}$ from $L^{p}\left(S_{\varepsilon}\right)$ into $L^{p}(\Omega \times S)$ is defined by

$$
\forall \phi \in L^{p}\left(S_{\varepsilon}\right), \quad \begin{cases}\mathcal{T}_{\varepsilon}^{b}(\phi)(x, y)=\phi(\varepsilon[x / \varepsilon]+\varepsilon y) & \text { for a.e. }(x, y) \in \widehat{\Omega}_{\varepsilon} \times S, \\ \mathcal{T}_{\varepsilon}^{b}(\phi)(x, y)=0 & \text { for a.e. }(x, y) \in \Lambda_{\varepsilon} \times S .\end{cases}
$$

Proposition 3.3. Assume $p \in[1,+\infty]$, then for every $\phi \in L^{p}\left(S_{\varepsilon}\right)$ one has

$$
\begin{aligned}
& \int_{\Omega \times S} \mathcal{T}_{\varepsilon}^{b}(\phi)(x, y) d x d \sigma_{y}=\varepsilon \int_{S_{\varepsilon}} \phi(x) d \sigma_{x}, \\
& \left\|\mathcal{T}_{\varepsilon}^{b}(\phi)\right\|_{L^{p}(\Omega \times S)}=\varepsilon^{1 / p}\|\phi\|_{L^{p}\left(S_{\varepsilon}\right)} .
\end{aligned}
$$

\footnotetext{
${ }^{1} \widetilde{H}^{1 / 2}(S)$ is the space of all $v \in H^{1 / 2}(S)$ whose extension by 0 in $\partial \mathcal{S} \backslash S$ belongs to $H^{1 / 2}(\partial \mathcal{S})($ see $[2])$.
} 


\subsection{Estimates in $L^{2}\left(\Omega ; H^{\alpha}(S)\right), \alpha \in(0,1)$.}

As immediate consequence of the definitions of the semi-norms in $H^{\alpha}(S)$ and $H^{\alpha}\left(S_{\varepsilon}\right)$, we have the following lemma and corollary:

Lemma 3.4. For every $u \in H^{\alpha}\left(S_{\varepsilon}\right), \alpha \in(0,1)$, one has

$$
\left\|\mathcal{T}_{\varepsilon}^{b}(u)\right\|_{L^{2}\left(\Omega ; H^{\alpha}(S)\right)}^{\prime}=\varepsilon^{1 / 2+\alpha}\|u\|_{H^{\alpha}\left(S_{\varepsilon}\right)}^{\prime} .
$$

Proof. One has

$$
\begin{aligned}
\left(\|u\|_{H^{\alpha}\left(S_{\varepsilon}\right)}^{\prime}\right)^{2} & =\sum_{\xi \in \Xi_{\varepsilon}} \int_{\varepsilon \xi+\varepsilon S} \int_{\varepsilon \xi+\varepsilon S} \frac{|u(x)-u(y)|^{2}}{|x-y|^{2+2 \alpha}} d \sigma_{x} d \sigma_{y} \\
& =\sum_{\xi \in \Xi_{\varepsilon}} \int_{S} \int_{S} \varepsilon^{4} \frac{|u(\varepsilon \xi+\varepsilon s)-u(\varepsilon \xi+\varepsilon t)|^{2}}{\varepsilon^{2+2 \alpha}|\xi+s-\xi-t|^{2+2 \alpha}} d \sigma_{s} d \sigma_{t} \\
& =\varepsilon^{-1-2 \alpha} \int_{\Omega} \int_{S} \int_{S} \frac{\left|\mathcal{T}_{\varepsilon}^{b}(u)(x, s)-\mathcal{T}_{\varepsilon}^{b}(u)(x, t)\right|^{2}}{|s-t|^{2+2 \alpha}} d \sigma_{s} d \sigma_{t} d x \\
& =\varepsilon^{-1-2 \alpha}\left(\left\|\mathcal{T}_{\varepsilon}^{b}(u)\right\|_{L^{2}\left(\Omega ; H^{\alpha}(S)\right)}^{\prime}\right)^{2}
\end{aligned}
$$

The equality is proved.

As immediate consequence of Propositions 3.3 and 3.4 one has

Corollary 3.5. For every $\phi \in H^{\alpha}\left(S_{\varepsilon}\right), \alpha \in(0,1)$, one has

$$
\left\|\left[\mathcal{T}_{\varepsilon}^{b}(\phi)\right]\right\|_{L^{2}\left(\Omega ; H^{\alpha}(S)\right)}=\varepsilon^{1 / 2}\|[\phi]\|_{H^{\alpha}\left(S_{\varepsilon}\right)} .
$$

For every $u \in H^{1}\left(\Omega_{\varepsilon}^{*}\right)^{3}$, one has

$$
\left\|\left[\mathcal{T}_{\varepsilon}^{b}(u)\right]_{S}\right\|_{L^{2}\left(\Omega ; H^{1 / 2}(S)\right)}=\varepsilon^{1 / 2}\left\|[u]_{S_{\varepsilon}}\right\|_{H^{1 / 2}\left(S_{\varepsilon}\right)} \leq C_{0} \varepsilon\|e(u)\|_{L^{2}\left(\Omega_{\varepsilon}^{*}\right)} .
$$

The constant does not depend on $\varepsilon$, it depends on the crack $S$.

Proof. Since the domain $\mathbf{Y}^{*}$ satisfies the Korn inequality, for every $v \in H^{1}\left(\mathbf{Y}^{*}\right)^{3}$ there exists a rigid displacement $r$ such that

$$
\|v-r\|_{H^{1}\left(\mathbf{Y}^{*}\right)} \leq C\left\|e_{y}(u)\right\|_{L^{2}\left(\mathbf{Y}^{*}\right)}
$$

The above inequality yields

$$
\left\|[v]_{S}\right\|_{L^{2}(S)}+\left\|[v]_{S}\right\|_{H^{1 / 2}(S)}^{\prime} \leq C\|v-r\|_{H^{1}\left(\mathbf{Y}^{*}\right)} \leq C_{0}\left\|e_{y}(v)\right\|_{L^{2}\left(\mathbf{Y}^{*}\right)}
$$

Applying the above estimate with $v=\mathcal{T}_{\varepsilon}^{*}(u)(\varepsilon \xi, \cdot), \xi \in \Xi_{\varepsilon}$, easily yields 3.7.

\subsection{Estimates for the displacements in $H_{\Gamma}^{1}\left(\Omega_{\varepsilon}^{*}\right)$, their traces and jumps}

Let $u$ be in $H_{\Gamma}^{1}\left(\Omega_{\varepsilon}^{*}\right)^{3}$ and $r_{u}(\varepsilon \xi, \cdot)$ the orthogonal projections of $u_{\mid \varepsilon \xi+\varepsilon \mathbf{Y}}, \xi \in \Xi_{\varepsilon}$, on $\mathcal{R}$,

$$
r_{u}(\varepsilon \xi, x)=a_{u}(\varepsilon \xi)+b_{u}(\varepsilon \xi) \wedge\left(\varepsilon\left\{\frac{x}{\varepsilon}\right\}\right), \quad x \in \varepsilon \xi+\varepsilon \mathbf{Y}, \quad a_{u}(\varepsilon \xi), b_{u}(\varepsilon \xi) \in \mathbb{R}^{3} .
$$

We start with the Korn inequality for the cracked domain.

Lemma 3.6. There exists a constant $C$ (independent of $\varepsilon$ ) such that for every $u \in H^{1}\left(\Omega_{\varepsilon}\right)^{3}$

$$
\begin{aligned}
& \sum_{\xi \in \Xi_{\varepsilon}}\left(\left\|u-r_{u}\right\|_{L^{2}\left(\varepsilon \xi+\varepsilon \mathbf{Y}^{*}\right)}^{2}+\varepsilon^{2}\left\|\nabla\left(u-r_{u}\right)\right\|_{L^{2}\left(\varepsilon \xi+\varepsilon \mathbf{Y}^{*}\right)}^{2}\right) \leq C \varepsilon^{2}\|e(u)\|_{L^{2}\left(\Omega_{\varepsilon}^{*}\right)}^{2}, \\
& \left\|u-r_{u}\right\|_{L^{2}\left(S_{\varepsilon}\right)} \leq C \sqrt{\varepsilon}\|e(u)\|_{L^{2}\left(\Omega_{\varepsilon}^{*}\right)}, \quad\left\|u-r_{u}\right\|_{H^{1 / 2}\left(S_{\varepsilon}\right)}^{\prime} \leq C\|e(u)\|_{L^{2}\left(\Omega_{\varepsilon}^{*}\right)} .
\end{aligned}
$$


Proof. Applying (3.4) (after $\varepsilon$-scaling) gives

$$
\left\|u-r_{u}\right\|_{L^{2}\left(\varepsilon \xi+\varepsilon \mathbf{Y}^{*}\right)}^{2}+\varepsilon^{2}\left\|\nabla\left(u-r_{u}\right)\right\|_{L^{2}\left(\varepsilon \xi+\varepsilon \mathbf{Y}^{*}\right)}^{2} \leq C \varepsilon^{2}\|e(u)\|_{L^{2}(\varepsilon \xi+\varepsilon \mathbf{Y})}^{2} .
$$

Then adding the above inequalities (with respect to $\xi$ ) yields $(3.9)_{1}$. Then one obtains $(3.9)_{2,3}$.

Next proposition provides the Korn inequality in terms of jumps and the estimate for jumps instead of traces.

Lemma 3.7. For every displacement $u$ in $H_{\Gamma}^{1}\left(\Omega_{\varepsilon}^{*}\right)^{3}$, one has

$$
\begin{aligned}
& \|u\|_{H^{1}\left(\Omega_{\varepsilon}^{*}\right)} \leq C\|e(u)\|_{L^{2}\left(\Omega_{\varepsilon}^{*}\right)}, \\
& \left\|[u]_{S_{\varepsilon}}\right\|_{H^{1 / 2}\left(S_{\varepsilon}\right)} \leq C_{0} \sqrt{\varepsilon}\|e(u)\|_{L^{2}\left(\Omega_{\varepsilon}^{*}\right)} .
\end{aligned}
$$

The constant does not depend on $\varepsilon$.

Proof. Proceeding as in [8], we obtain the existence of a constant independent of $\varepsilon$ such that $(3.10)_{1}$ is satisfied. Then 3.10$)_{2}$ is given by (3.7).

\section{The unfolding operator from $H^{-\alpha}\left(S_{\varepsilon}\right)$ into $L^{2}\left(\Omega ; H^{-\alpha}(S)\right), \alpha \in(0,1)$}

Definition 2. For $p \in[1,+\infty]$, the averaging boundary operator $\mathcal{U}_{\varepsilon}^{b}: L^{p}(\Omega \times S) \longmapsto L^{p}\left(S_{\varepsilon}\right)$ is defined by

$$
\forall \Phi \in L^{p}(\Omega \times S), \quad \mathcal{U}_{\varepsilon}^{b}(\Phi)(x)= \begin{cases}\int_{Y} \Phi\left(\varepsilon\left[\frac{x}{\varepsilon}\right]+\varepsilon z,\left\{\frac{x}{\varepsilon}\right\}\right) d z & \text { for a.e. } x \in \widehat{\Omega}_{\varepsilon}, \\ 0 & \text { for a.e. } x \in \Lambda_{\varepsilon}\end{cases}
$$

\subsubsection{Some properties involving $\mathcal{U}_{\varepsilon}^{b}$ and $\mathcal{T}_{\varepsilon}^{b}$}

Let $p$ be in $[1,+\infty], p^{\prime}$ its conjugate $\left(1 / p+1 / p^{\prime}=1\right)$

- $\forall \Phi \in L^{p}(\Omega \times S), \quad \forall \psi \in L^{p^{\prime}}\left(S_{\varepsilon}\right), \int_{S_{\varepsilon}} \psi \mathcal{U}_{\varepsilon}^{b}(\Phi) d \sigma=\frac{1}{\varepsilon} \int_{\Omega \times S} \mathcal{T}_{\varepsilon}^{b}(\psi) \Phi d x d \sigma_{y}$

- $\mathcal{U}_{\varepsilon}^{b}$ is almost a right inverse of $\mathcal{T}_{\varepsilon}^{b}$

$\forall \Phi \in L^{p}(\Omega \times S), \quad \mathcal{T}_{\varepsilon}^{b} \circ \mathcal{U}_{\varepsilon}^{b}(\Phi)(x, y)= \begin{cases}\int_{Y} \Phi\left(\varepsilon\left[\frac{x}{\varepsilon}\right]+\varepsilon z, y\right) d z & \text { for a.e. for }(x, y) \in \widehat{\Omega}_{\varepsilon} \times S, \\ 0 & \text { for a.e. }(x, y) \in \Lambda_{\varepsilon} \times S .\end{cases}$

- $\mathcal{U}_{\varepsilon}^{b}$ is a left inverse of $\mathcal{T}_{\varepsilon}^{b}$

$$
\forall \phi \in L^{p}\left(S_{\varepsilon}\right), \quad \mathcal{U}_{\varepsilon}^{b} \circ \mathcal{T}_{\varepsilon}^{b}(\phi)=\phi,
$$

- for every $\Phi$ in $L^{2}\left(\Omega ; H^{\alpha}(S)\right)$ one has $\mathcal{U}_{\varepsilon}^{b}(\Phi) \in H^{\alpha}\left(S_{\varepsilon}\right)$.

For every $\alpha \in(0,1)$, denote $H^{-\alpha}\left(S_{\varepsilon}\right)$ (resp. $\left.\widetilde{H}^{-\alpha}\left(S_{\varepsilon}\right)\right)$ the dual space of $H^{\alpha}\left(S_{\varepsilon}\right)$ (resp. $\widetilde{H}^{\alpha}\left(S_{\varepsilon}\right)$ ) equipped with the dual norm.

Now, for $g \in H^{-\alpha}\left(S_{\varepsilon}\right)$ (resp. $\left.\widetilde{H}^{-\alpha}\left(S_{\varepsilon}\right)\right), \alpha \in(0,1)$, one defines by duality $\mathcal{T}_{\varepsilon}^{b}(g) \in L^{2}\left(\Omega ; H^{-\alpha}(S)\right.$ ) (resp. $\left.\mathcal{T}_{\varepsilon}^{b}(g) \in L^{2}\left(\Omega ; \widetilde{H}^{-\alpha}(S)\right)\right)$ as

$$
\begin{aligned}
\left\langle\mathcal{T}_{\varepsilon}^{b}(g), \Phi\right\rangle_{L^{2}\left(\Omega ; H^{-\alpha}(S)\right), L^{2}\left(\Omega ; H^{\alpha}(S)\right)} & \doteq \varepsilon\left\langle g, \mathcal{U}_{\varepsilon}^{b}(\Phi)\right\rangle_{H^{-\alpha}\left(S_{\varepsilon}\right), H^{\alpha}\left(S_{\varepsilon}\right)} & \forall \Phi \in L^{2}\left(\Omega ; H^{\alpha}(S)\right), \\
\left(\operatorname{resp} .\left\langle\mathcal{T}_{\varepsilon}^{b}(g), \Phi\right\rangle_{L^{2}\left(\Omega ; \widetilde{H}^{-\alpha}(S)\right), L^{2}\left(\Omega ; \widetilde{H}^{\alpha}(S)\right)}\right. & \doteq \varepsilon\left\langle g, \mathcal{U}_{\varepsilon}^{b}(\Phi)\right\rangle_{\widetilde{H}^{-\alpha}\left(S_{\varepsilon}\right), \widetilde{H}^{\alpha}\left(S_{\varepsilon}\right)} & \left.\forall \Phi \in L^{2}\left(\Omega ; \widetilde{H}^{\alpha}(S)\right)\right)
\end{aligned}
$$


Observe that

$$
\begin{array}{rlrl}
\left\langle\mathcal{T}_{\varepsilon}^{b}(g), \mathcal{T}_{\varepsilon}^{b}(\phi)\right\rangle_{L^{2}\left(\Omega ; H^{-\alpha}(S)\right), L^{2}\left(\Omega ; H^{\alpha}(S)\right)} & =\varepsilon\langle g, \phi\rangle_{H^{-\alpha}\left(S_{\varepsilon}\right), H^{\alpha}\left(S_{\varepsilon}\right)} & \forall \phi \in H^{\alpha}\left(S_{\varepsilon}\right), \\
\left(\operatorname{resp.}\left\langle\mathcal{T}_{\varepsilon}^{b}(g), \mathcal{T}_{\varepsilon}^{b}(\phi)\right\rangle_{L^{2}\left(\Omega ; \widetilde{H}^{-\alpha}(S)\right), L^{2}\left(\Omega ; \widetilde{H}^{\alpha}(S)\right)}=\varepsilon\langle g, \phi\rangle_{\widetilde{H}^{-\alpha}\left(S_{\varepsilon}\right), \widetilde{H}^{\alpha}\left(S_{\varepsilon}\right)}\right. & \left.\forall \phi \in \widetilde{H}^{\alpha}\left(S_{\varepsilon}\right)\right)
\end{array}
$$

since here

$$
\begin{aligned}
& \forall \phi \in H^{\alpha}\left(S_{\varepsilon}\right), \quad \mathcal{U}_{\varepsilon}^{b} \circ \mathcal{T}_{\varepsilon}^{*}(\phi)=\phi, \\
& \text { (resp. } \forall \phi \in \widetilde{H}^{\alpha}\left(S_{\varepsilon}\right), \quad \mathcal{U}_{\varepsilon}^{b} \circ \mathcal{T}_{\varepsilon}^{*}(\phi)=\phi \text { ) }
\end{aligned}
$$

Lemma 4.1. For every $\alpha \in(0,1)$, one has

$$
\begin{aligned}
\left\|\mathcal{T}_{\varepsilon}^{b}(\phi)\right\|_{L^{2}\left(\Omega ; H^{-\alpha}(S)\right)} & =\varepsilon^{1 / 2}\|\phi\|_{H^{-\alpha}\left(S_{\varepsilon}\right)}, & & \forall \phi \in H^{-\alpha}\left(S_{\varepsilon}\right), \\
\left\|\mathcal{T}_{\varepsilon}^{b}(\phi)\right\|_{L^{2}\left(\Omega ; \widetilde{H}^{-\alpha}(S)\right)} & =\varepsilon^{1 / 2}\|\phi\|_{\widetilde{H}^{-\alpha}\left(S_{\varepsilon}\right)}, & & \forall \phi \in \widetilde{H}^{-\alpha}\left(S_{\varepsilon}\right) .
\end{aligned}
$$

Proof. One proves 4.4$)_{1}$, the proof of 4.4$)_{2}$ is obtained following the same lines. One has

$$
\forall \Phi \in L^{2}\left(\Omega ; H^{\alpha}(S)\right), \quad\left\langle\mathcal{T}_{\varepsilon}^{b}(g), \Phi\right\rangle_{L^{2}\left(\Omega ; H^{-\alpha}(S)\right), L^{2}\left(\Omega ; H^{\alpha}(S)\right)} \leq \varepsilon\|g\|_{H^{-\alpha}\left(S_{\varepsilon}\right)}\left\|\mathcal{U}_{\varepsilon}^{b}(\Phi)\right\|_{H^{\alpha}\left(S_{\varepsilon}\right)} .
$$

Then, Property 4.1) and Propositions 3.3 3.4 lead to

$$
\begin{gathered}
\left\|\mathcal{U}_{\varepsilon}^{b}(\Phi)\right\|_{L^{2}\left(S_{\varepsilon}\right)}=\varepsilon^{-1 / 2}\left\|\mathcal{T}_{\varepsilon}^{b} \circ \mathcal{U}_{\varepsilon}^{b}(\Phi)\right\|_{L^{2}(\Omega \times)} \leq \varepsilon^{-1 / 2}\|\Phi\|_{L^{2}(\Omega \times S)}, \\
\varepsilon^{\alpha}\left\|\mathcal{U}_{\varepsilon}^{b}(\Phi)\right\|_{H^{\alpha}\left(S_{\varepsilon}\right)}^{\prime}=\varepsilon^{-1 / 2}\left\|\mathcal{T}_{\varepsilon}^{b} \circ \mathcal{U}_{\varepsilon}^{b}(\Phi)\right\|_{L^{2}\left(\Omega ; H^{\alpha}(S)\right)}^{\prime} \leq \varepsilon^{-1 / 2}\|\Phi\|_{L^{2}\left(\Omega ; H^{\alpha}(S)\right)}^{\prime}
\end{gathered}
$$

Hence

$$
\forall \Phi \in L^{2}\left(\Omega ; H^{\alpha}(S)\right), \quad\left\langle\mathcal{T}_{\varepsilon}^{b}(g), \Phi\right\rangle_{L^{2}\left(\Omega ; H^{-\alpha}(S)\right), L^{2}\left(\Omega ; H^{\alpha}(S)\right)} \leq \varepsilon^{1 / 2}\|g\|_{H^{-\alpha}\left(S_{\varepsilon}\right)}\|\Phi\|_{L^{2}\left(\Omega ; H^{\alpha}(S)\right)} .
$$

which yields $\left\|\mathcal{T}_{\varepsilon}^{b}(g)\right\|_{L^{2}\left(\Omega ; H^{-\alpha}(S)\right)} \leq \varepsilon^{1 / 2}\|g\|_{H^{-\alpha}\left(S_{\varepsilon}\right)}$.

Now, due to the Property 4.2, for every $\phi \in H^{\alpha}\left(S_{\varepsilon}\right)$ one has

$$
\langle g, \phi\rangle_{H^{-\alpha}\left(S_{\varepsilon}\right), H^{\alpha}\left(S_{\varepsilon}\right)}=\frac{1}{\varepsilon}\left\langle\mathcal{T}_{\varepsilon}^{b}(g), \mathcal{T}_{\varepsilon}^{b}(\phi)\right\rangle_{L^{2}\left(\Omega ; H^{-\alpha}(S)\right), L^{2}\left(\Omega ; H^{\alpha}(S)\right)} .
$$

So

$$
\langle g, \phi\rangle_{H^{-\alpha}\left(S_{\varepsilon}\right), H^{\alpha}\left(S_{\varepsilon}\right)} \leq \frac{1}{\varepsilon}\left\|\mathcal{T}_{\varepsilon}^{b}(g)\right\|_{L^{2}\left(\Omega ; H^{-\alpha}(S)\right)}\left\|\mathcal{T}_{\varepsilon}^{b}(\phi)\right\|_{L^{2}\left(\Omega ; H^{\alpha}(S)\right)} .
$$

The estimates in Propositions 3.3 3.4 yield $\left\|\mathcal{T}_{\varepsilon}^{b}(\phi)\right\|_{L^{2}\left(\Omega ; H^{\alpha}(S)\right)} \leq \varepsilon^{1 / 2}\|\phi\|_{H^{\alpha}\left(S_{\varepsilon}\right)}$, hence

$$
\|g\|_{H^{-\alpha}\left(S_{\varepsilon}\right)} \leq \varepsilon^{-1 / 2}\left\|\mathcal{T}_{\varepsilon}^{b}(g)\right\|_{L^{2}\left(\Omega ; H^{-\alpha}(S)\right)} .
$$

The lemma is proved.

Lemma 4.2. Let $h_{\varepsilon}$ be in $L^{2}\left(\mathcal{S}_{\varepsilon}\right)$ and $v_{\varepsilon}$ be a field in $L^{2}\left(\mathcal{S}_{\varepsilon}\right)^{3}$ such that

$$
\operatorname{div}\left(v_{\varepsilon}\right)=h_{\varepsilon} \quad \text { in } \quad \mathcal{D}^{\prime}\left(\mathcal{S}_{\varepsilon}\right) .
$$

One has

$$
\left\|v_{\varepsilon} \cdot \nu_{\varepsilon}\right\|_{H^{-1 / 2}\left(\partial \mathcal{S}_{\varepsilon}\right)} \leq C\left(\varepsilon\left\|h_{\varepsilon}\right\|_{L^{2}\left(\mathcal{S}_{\varepsilon}\right)}+\left\|v_{\varepsilon}\right\|_{L^{2}\left(\mathcal{S}_{\varepsilon}\right)}\right) .
$$

The constant does not depend on $\varepsilon$.

Proof. Due to the hypothesis of the lemma, one has for every $\xi \in \Xi_{\varepsilon}$

$$
\mathcal{T}_{\varepsilon}^{b}\left(v_{\varepsilon}\right)(\varepsilon \xi, \cdot) \cdot \nu \in H^{-1 / 2}(\partial \mathcal{S}), \quad \operatorname{div}_{y}\left(\mathcal{T}_{\varepsilon}^{*}\left(v_{\varepsilon}\right)\right)=\varepsilon \mathcal{T}_{\varepsilon}^{*}\left(h_{\varepsilon}\right) \quad \text { in } \quad \mathcal{D}^{\prime}(\Omega \times \mathcal{S})
$$

and the estimate

$$
\left\|\mathcal{T}_{\varepsilon}^{b}\left(v_{\varepsilon}\right)(\varepsilon \xi, \cdot) \cdot \nu\right\|_{H^{-1 / 2}(\partial \mathcal{S})}^{2} \leq C\left(\varepsilon^{2}\left\|\mathcal{T}_{\varepsilon}\left(h_{\varepsilon}\right)(\varepsilon \xi, \cdot)\right\|_{L^{2}(\mathcal{S})}^{2}+\left\|\mathcal{T}_{\varepsilon}\left(v_{\varepsilon}\right)(\varepsilon \xi, \cdot)\right\|_{L^{2}(\mathcal{S})}^{2}\right) .
$$

Adding the above inequalities for $\xi \in \Xi_{\varepsilon}$ yields

$$
\left\|\mathcal{T}_{\varepsilon}^{b}\left(v_{\varepsilon}\right) \cdot \nu\right\|_{L^{2}\left(\Omega ; H^{-1 / 2}(\partial \mathcal{S})\right)}^{2} \leq C\left(\varepsilon^{2}\left\|\mathcal{T}_{\varepsilon}^{*}\left(h_{\varepsilon}\right)\right\|_{L^{2}(\Omega \times \mathcal{S})}^{2}+\left\|\mathcal{T}_{\varepsilon}^{*}\left(v_{\varepsilon}\right)\right\|_{L^{2}(\Omega \times \mathcal{S})}^{2}\right)
$$

which in turn with Proposition 3.1 give 4.6 . 


\section{Statement of the contact $\varepsilon$-problem with Coulomb's friction on periodic cracks}

Assume that one has a given symmetric bilinear form on $H_{\Gamma}^{1}\left(\Omega_{\varepsilon}^{*}\right)^{3}$

$$
\mathbf{a}^{\varepsilon}(u, v) \doteq \int_{\Omega_{\varepsilon}^{*}} a_{i j k l}^{\varepsilon}(x) e_{i j}(u)(x) e_{k l}(v)(x) d x, \quad e_{i j}(u)=\frac{1}{2}\left(\frac{\partial u_{i}}{\partial x_{j}}+\frac{\partial u_{j}}{\partial x_{j}}\right), \quad(i, j) \in\{1,2,3\}^{2}
$$

where the tensor field $a^{\varepsilon}=\left(a_{i j k l}^{\varepsilon}\right), a_{i j k l}^{\varepsilon} \in L^{\infty}\left(\Omega_{\varepsilon}^{*}\right)$, has the usual properties of symmetry, boundedness and coercivity when operating on symmetric $3 \times 3$ matrices

$$
a_{i j k l}^{\varepsilon}=a_{j i k l}^{\varepsilon}=a_{k l i j}^{\varepsilon}, \quad \bar{\alpha} \eta_{i j} \eta_{i j} \leq a_{i j k l}^{\varepsilon} \eta_{i j} \eta_{k l} \leq C_{A} \eta_{i j} \eta_{k l} \quad \text { a.e. in } \in \Omega_{\varepsilon}^{*} .
$$

The vector fields $v$ are the admissible displacement fields with respect to the reference configuration $\Omega_{\varepsilon}^{*}$. The tensor field

$$
\sigma_{k l}^{\varepsilon}(v) \doteq a_{i j k l}^{\varepsilon} e_{i j}(v) \quad \forall v \in H^{1}\left(\Omega_{\varepsilon}^{*}\right)^{3}
$$

is the stress tensor associated to the strain tensor $e(v)$.

\section{Assumptions}

- The functions $a_{i j k l}$ belong to $L^{\infty}\left(\mathbf{Y}^{*}\right)$ and they are $W^{1, \infty}$ in a neighborhood of $S, a_{i j k l}^{\varepsilon}=a_{i j k l}\left(\left\{\frac{\cdot}{\varepsilon}\right\}\right)$ a.e. in $\mathbb{R}^{3}$,

- the applied volume forces $f$ belong to $L^{2}(\Omega)^{3}$,

- the friction coefficient $\mu$ is a non-negative function belonging to $W^{1, \infty}(\Omega)$ with support included in $\Omega^{\prime} \subset \Omega:^{2}$

- $\mathcal{K}_{\varepsilon}$ is a convex set defined by

$$
\mathcal{K}_{\varepsilon} \doteq\left\{v \in H_{\Gamma}^{1}\left(\Omega_{\varepsilon}^{*}\right)^{3} \mid\left[v_{\nu_{\varepsilon}}\right]_{S_{\varepsilon}} \leq 0\right\} .
$$

The vector fields $v \in \mathcal{K}_{\varepsilon}$ are the admissible displacement fields with respect to the reference configuration $\Omega_{\varepsilon}^{*}$. The inequality in the definition of $\mathcal{K}_{\varepsilon}$ represent the non-penetration condition.

The strong formulation of the static contact problem is the following:

$$
\left\{\begin{array}{l}
\text { Find } u_{\varepsilon} \text { in } \mathcal{K}_{\varepsilon} \text { such that, } \\
-\operatorname{div} \sigma_{\varepsilon}\left(u_{\varepsilon}\right)=f \text { in } \Omega_{\varepsilon}^{*}, \\
\sigma_{\nu_{\varepsilon}}\left(u_{\varepsilon}\right)\left[\left(u_{\varepsilon}\right)_{\nu_{\varepsilon}}\right]_{S_{\varepsilon}}=0,\left.\quad \sigma_{\nu_{\varepsilon}}\left(u_{\varepsilon}\right)\right|_{S_{\varepsilon}} \leq 0, \quad\left[\sigma_{\nu_{\varepsilon}}\left(u_{\varepsilon}\right)\right]_{S_{\varepsilon}}=0, \\
\left|\sigma_{\tau_{\varepsilon}}\left(u_{\varepsilon}\right)\right|<\mu\left|\sigma_{\nu_{\varepsilon}}\left(u_{\varepsilon}\right)\right| \Rightarrow\left[\left(u_{\varepsilon}\right)_{\tau_{\varepsilon}}\right]_{S_{\varepsilon}}=0 \quad \text { on } S_{\varepsilon}, \\
\sigma_{\tau_{\varepsilon}}\left(u_{\varepsilon}\right)=-\mu\left|\sigma_{\nu_{\varepsilon}}\left(u_{\varepsilon}\right)\right| \frac{\left[\left(u_{\varepsilon}\right)_{\tau_{\varepsilon}}\right]_{S_{\varepsilon}}}{\left|\left[\left(u_{\varepsilon}\right)_{\tau_{\varepsilon}}\right]\right|_{S_{\varepsilon}}} \Rightarrow\left[\left(u_{\varepsilon}\right)_{\tau_{\varepsilon}}\right]_{S_{\varepsilon}} \neq 0 \quad \text { on } S_{\varepsilon}, \\
\sigma_{\varepsilon}\left(u_{\varepsilon}\right) \cdot \nu=0 \text { on } \partial \Omega \backslash \Gamma, \\
u_{\varepsilon}=0 \text { on } \Gamma,
\end{array}\right.
$$

where $\sigma_{\nu_{\varepsilon}}(v)=\left(\sigma_{\varepsilon}(v) \nu_{\varepsilon}\right) \cdot \nu_{\varepsilon}, \sigma_{\tau_{\varepsilon}}(v)=\sigma_{\varepsilon}(v) \nu_{\varepsilon}-\sigma_{\nu_{\varepsilon}}(v) \nu_{\varepsilon}$.

The weak formulation of the static contact problem is

Find $U_{\varepsilon} \in \mathcal{K}_{\varepsilon}$ such that for every $v \in \mathcal{K}_{\varepsilon}$,

$$
\mathbf{a}^{\varepsilon}\left(U_{\varepsilon}, v-U_{\varepsilon}\right)+\left\langle\mu\left|\sigma_{\nu_{\varepsilon}}\left(U_{\varepsilon}\right)\right|,\left|\left[v_{\tau_{\varepsilon}}\right]_{S_{\varepsilon}}\right|-\left|\left[\left(U_{\varepsilon}\right)_{\tau_{\varepsilon}}\right]_{S_{\varepsilon}}\right|\right\rangle_{\varepsilon} \geq\left(f, v-U_{\varepsilon}\right)
$$

\footnotetext{
${ }^{2}$ with a few change, we can choose a friction coefficient in the form $\mu_{\varepsilon}(x)=\mu\left(x,\left\{\frac{x}{\varepsilon}\right\}\right)$ for a.e. $x \in \Omega^{\prime} \times S$ where $\mu \in W^{1, \infty}(\Omega \times S)$ vanishes in $\left.\left(\Omega \backslash \overline{\Omega^{\prime}}\right) \times S\right)$.
} 
where

$$
\begin{aligned}
& \forall(g, w) \in \widetilde{H}^{-1 / 2}\left(S_{\varepsilon}\right) \times \widetilde{H}^{1 / 2}\left(S_{\varepsilon}\right), \quad\langle g, w\rangle_{\varepsilon}=\langle g, w\rangle_{\widetilde{H}^{-1 / 2}\left(S_{\varepsilon}\right), \widetilde{H}^{1 / 2}\left(S_{\varepsilon}\right)}, \\
& \forall v \in L^{2}\left(\Omega_{\varepsilon}^{*}\right)^{3}, \quad(f, v)=\int_{\Omega_{\varepsilon}^{*}} f \cdot v d x .
\end{aligned}
$$

To solve the above problem, we consider the contact problem with given friction

$$
\left\{\begin{array}{l}
\text { Find } U_{\varepsilon, G} \in \mathcal{K}_{\varepsilon} \text { such that for every } v \in \mathcal{K}_{\varepsilon}, \\
\mathbf{a}^{\varepsilon}\left(U_{\varepsilon, G}, v-U_{\varepsilon, G}\right)+\left\langle G,\left|\left[v_{\tau_{\varepsilon}}\right]_{S_{\varepsilon}}\right|-\left|\left[\left(U_{\varepsilon, G}\right)_{\tau_{\varepsilon}}\right]_{S_{\varepsilon}}\right|\right\rangle_{\varepsilon} \geq\left(f, v-U_{\varepsilon, G}\right)
\end{array}\right.
$$

where the linear form $G$ is an element of the cone

$$
\mathcal{C}_{\varepsilon}^{*} \doteq\left\{G \in \widetilde{H}^{-1 / 2}\left(S_{\varepsilon}\right) \mid \forall \phi \in \widetilde{H}^{1 / 2}\left(S_{\varepsilon}\right), \quad \phi \geq 0 \Longrightarrow\langle G, \phi\rangle_{\varepsilon} \geq 0\right\} .
$$

The existence and uniqueness of the solution of problem $(5.3)$ is obtained as the solution of a minimization convex functional (see Section 6).

\subsection{Estimates}

One has the following estimates:

Proposition 5.1. The solution $U_{\varepsilon, G}$ of the contact problem 5.3 satisfies the following a priori estimates:

$$
\begin{aligned}
& \left\|U_{\varepsilon, G}\right\|_{H^{1}\left(\Omega_{\varepsilon}^{*}\right)} \leq C\|f\|_{L^{2}(\Omega)}, \\
& \left\|\left[U_{\varepsilon, G}\right]_{S_{\varepsilon}}\right\|_{H^{1 / 2}\left(S_{\varepsilon}\right)} \leq C_{0} \sqrt{\varepsilon}\|f\|_{L^{2}(\Omega)} .
\end{aligned}
$$

Furthermore, one has

$$
\sigma_{\varepsilon}\left(U_{\varepsilon, G}\right)_{\mid \partial \mathcal{S}_{\varepsilon}^{-}} \nu_{\varepsilon}=\sigma_{\varepsilon}\left(U_{\varepsilon, G}\right)_{\mid \partial \mathcal{S}_{\varepsilon}^{+}} \nu_{\varepsilon} \quad \text { in } \quad \widetilde{H}^{-1 / 2}\left(S_{\varepsilon}\right)^{3}
$$

and

$$
\sqrt{\varepsilon}\left\|\sigma_{\nu_{\varepsilon}}\left(U_{\varepsilon, G}\right)\right\|_{H^{-1 / 2}\left(S_{\varepsilon}\right)} \leq C\|f\|_{L^{2}(\Omega)}
$$

where $\sigma_{\nu_{\varepsilon}}\left(U_{\varepsilon, G}\right) \doteq\left(\sigma_{\varepsilon}\left(U_{\varepsilon, G}\right)_{\mid \partial \mathcal{S}_{\varepsilon}^{+}} \nu_{\varepsilon}\right) \cdot \nu_{\varepsilon}$ and $-\sigma_{\nu_{\varepsilon}}\left(U_{\varepsilon, G}\right)$ belongs to $\mathcal{C}_{\varepsilon}^{*}$.

The constants do not depend on $\varepsilon$.

Proof. From inequality (5.3), one obtains

$$
\mathbf{a}^{\varepsilon}\left(U_{\varepsilon, G}, U_{\varepsilon, G}\right)+\left\langle G,\left|\left[\left(U_{\varepsilon, G}\right)_{\tau_{\varepsilon}}\right]_{S_{\varepsilon}}\right|\right\rangle_{\varepsilon} \leq\left(f, U_{\varepsilon, G}\right) .
$$

Then estimates (5.4) follow the ones of Lemma 3.7.

Now, let $v$ be in $H_{\Gamma}^{1}(\Omega)^{3}$. Since $\left[v_{\nu_{\varepsilon}}\right]_{S_{\varepsilon}}=0$, the fields $U_{\varepsilon, G}+v$ and $U_{\varepsilon, G}-v$ belong to $\mathcal{K}_{\varepsilon}$, one has

$$
\mathbf{a}^{\varepsilon}\left(U_{\varepsilon, G}, U_{\varepsilon, G} \pm v-U_{\varepsilon, G}\right)+\left\langle G,\left|\left[\left(U_{\varepsilon, G} \pm v\right)_{\tau_{\varepsilon}}\right]\right|_{S_{\varepsilon}}-\left|\left[\left(U_{\varepsilon, G}\right)_{\tau_{\varepsilon}}\right]\right|_{S_{\varepsilon}}\right\rangle_{\varepsilon} \geq(f, \pm v)
$$

Since $v$ belongs to $H_{\Gamma}^{1}(\Omega)^{3}$

$$
\left[\left(U_{\varepsilon, G} \pm v\right)_{\tau_{\varepsilon}}\right]_{S_{\varepsilon}}=\left[\left(U_{\varepsilon, G}\right)_{\tau_{\varepsilon}}\right]_{S_{\varepsilon}}
$$

Hence

$$
\mathbf{a}^{\varepsilon}\left(U_{\varepsilon, G}, v\right) \geq(f, v) \text { and } \mathbf{a}^{\varepsilon}\left(U_{\varepsilon, G},-v\right) \geq(f,-v) .
$$

As a consequence

$$
\forall v \in H_{\Gamma}^{1}(\Omega)^{3}, \quad \mathbf{a}^{\varepsilon}\left(U_{\varepsilon, G}, v\right)=(f, v)
$$

which gives (5.5). Due to the estimates (4.6)-(5.4) 1 and the fact that $\operatorname{div}\left(\sigma_{\varepsilon}\left(U_{\varepsilon, G}\right)\right)=f$ in $\mathcal{S}_{\varepsilon}$ we obtain (5.6). To prove that $-\sigma_{\nu_{\varepsilon}}\left(U_{\varepsilon, G}\right)$ belongs to $\mathcal{C}_{\varepsilon}^{*}$ consider a non-negative function $\phi \in H^{1 / 2}(\partial \mathcal{S})$ vanishing in $\partial \mathcal{S} \backslash S$. 
Define $v$ as a lifting (vanishing on the boundary of $\mathbf{Y}$ ) of $\phi \nu$ in $H^{1}(\mathbf{Y} \backslash \overline{\mathcal{S}})^{3}$ and by 0 in $\mathcal{S}$. By construction $v \in H^{1}\left(\mathbf{Y}^{*}\right)^{3}$ and

$$
\left[v_{\tau}\right]_{S}=0, \quad\left(v_{\nu}\right)_{\mid \partial \mathcal{S}^{+}}=\phi, \quad\left(v_{\nu}\right)_{\mid \partial \mathcal{S}^{-}}=0, \quad\left[v_{\nu}\right]_{S}=\phi
$$

For $\xi$ every $\Xi_{\varepsilon}$, set

$$
v_{\varepsilon}^{\xi}=\left\{\begin{array}{lll}
\varepsilon v\left(\left\{\frac{\cdot}{\varepsilon}\right\}\right) & \text { a.e. in } & \varepsilon \xi+\varepsilon \mathbf{Y}, \\
0 & \text { a.e. in } & \Omega \backslash(\varepsilon \xi+\varepsilon \overline{\mathbf{Y}}) .
\end{array}\right.
$$

Taking $U_{\varepsilon, G}-v_{\varepsilon}^{\xi}$ as test displacement in $(5.3)$ and using the fact that $\operatorname{div}\left(\sigma_{\varepsilon}\left(U_{\varepsilon, G}\right)\right)=f$ in $\Omega_{\varepsilon}^{*}$, one obtains that $-\sigma_{\nu_{\varepsilon}}\left(U_{\varepsilon, G}\right) \in \mathcal{C}_{\varepsilon}^{*}$.

\section{Regularized Coulomb friction problem}

In order to prove the existence of solutions to the problem $(5.2)$, under a suitable assumption on the boundary of $\mathcal{S}$, one can show (as in [4]) that there exists $\alpha \in(0,1 / 2)$ such that

$$
\forall G \in \mathcal{C}_{\varepsilon}^{*} \cap H^{-1 / 2+\alpha}\left(S_{\varepsilon}\right), \quad \mu\left|\sigma_{\nu_{\varepsilon}}\left(U_{\varepsilon, G}\right)\right| \in \mathcal{C}_{\varepsilon}^{*} \cap H^{-1 / 2+\alpha}\left(S_{\varepsilon}\right) \text { and } \sqrt{\varepsilon}\left\|\sigma_{\nu_{\varepsilon}}\left(U_{\varepsilon, G}\right)\right\|_{H^{-1 / 2+\alpha}\left(S_{\varepsilon}\right)} \leq C\|f\|_{L^{2}(\Omega)} .
$$

Then, the Schauder's theorem (see Theorem 8.3) gives the existence of fixed points for the map

$$
A: G \in \mathcal{C}_{\varepsilon}^{*} \cap H^{-1 / 2+\alpha}\left(S_{\varepsilon}\right) \longmapsto \mu\left|\sigma_{\nu_{\varepsilon}}\left(U_{\varepsilon, G}\right)\right| \in \mathcal{C}_{\varepsilon}^{*} \cap H^{-1 / 2+\alpha}\left(S_{\varepsilon}\right) .
$$

Thus, the problem (5.2) admits solutions. Estimate 4.4 yields

$$
\left\|\mathcal{T}_{\varepsilon}^{b}\left(\sigma_{\nu_{\varepsilon}}\left(U_{\varepsilon, G}\right)\right)\right\|_{L^{2}\left(\Omega ; \widetilde{H}^{-1 / 2+\alpha}(S)\right)}=\varepsilon^{1 / 2}\left\|\sigma_{\nu_{\varepsilon}}\left(U_{\varepsilon, G}\right)\right\|_{\widetilde{H}^{-1 / 2+\alpha}\left(S_{\varepsilon}\right)} \leq C\|f\|_{L^{2}(\Omega)}
$$

For the homogenization process we need the compactness of the sequence $\left\{\mathcal{T}_{\varepsilon}^{b}\left(\sigma_{\nu_{\varepsilon}}\left(U_{\varepsilon, G}\right)\right)\right\}_{\varepsilon}$ in $L^{2}\left(\Omega ; \widetilde{H}^{-1 / 2}(S)\right)$. Unfortunately, the above estimate is not sufficient. One must improve (5.6), this could be obtain by comparing the norms of the tangential jumps $\left|\left[\left(U_{\varepsilon, G}\right)_{\tau_{\varepsilon}}\right]_{S_{\varepsilon}}\right|$ in two neighboring cells. But, it is well known that the following inequality:

$$
\||u|-|v|\|_{H^{1 / 2}\left(S_{\varepsilon}\right)} \leq C\|u-v\|_{H^{1 / 2}\left(S_{\varepsilon}\right)}, \quad \forall(u, v) \in H^{1}\left(S_{\varepsilon}\right)^{3} \times H^{1}\left(S_{\varepsilon}\right)^{3}
$$

is false. Moreover, one can not replace the euclidian norm $|\cdot|$ by any kind of its approximation because, if one has

$$
\|f(u)-f(v)\|_{H^{1 / 2}\left(S_{\varepsilon}\right)} \leq C\|u-v\|_{H^{1 / 2}\left(S_{\varepsilon}\right)}, \quad \forall(u, v) \in H^{1}\left(S_{\varepsilon}\right)^{3} \times H^{1}\left(S_{\varepsilon}\right)^{3}
$$

with e.g. $f \in \mathcal{C}^{1}\left(\mathbb{R}^{3} ; \mathbb{R}^{3}\right)$ then $f$ is affine!

On the basis of Proposition 10.1 (see Annex 10) and in order to perform the homogenization process, we choose to modify the problem (5.2) by adding a regularization term.

We equip $H_{\Gamma}^{2}\left(\Omega_{\varepsilon}^{*}\right)^{3}$ with the following semi-norm:

$$
\mathbf{N}_{\varepsilon}(u) \doteq \sqrt{\|e(u)\|_{L^{2}\left(\Omega_{\varepsilon}^{*}\right)}^{2}+\varepsilon^{2}\|\nabla e(u)\|_{L^{2}\left(\Omega_{\varepsilon}^{*}\right)}^{2}}
$$

Since the displacements belonging to $H_{\Gamma}^{2}\left(\Omega_{\varepsilon}^{*}\right)^{3}$ vanish on $\Gamma$, this semi-norm is a norm and thus $H_{\Gamma}^{2}\left(\Omega_{\varepsilon}^{*}\right)^{3}$ is a Hilbert space.

In view of Proposition 5.1, denote

$$
\mathbf{H}_{\varepsilon}(\Omega) \doteq\left\{u \in H_{\Gamma}^{2}\left(\Omega_{\varepsilon}^{*}\right)^{3} \mid \quad\left(\sigma^{\varepsilon}(u)_{\mid \partial \mathcal{S}_{\varepsilon}^{-}} \nu_{\varepsilon}\right) \cdot \nu_{\varepsilon}=\left(\sigma^{\varepsilon}(u)_{\mid \partial \mathcal{S}_{\varepsilon}^{+}} \nu_{\varepsilon}\right) \cdot \nu_{\varepsilon} \leq 0 \text { a.e. in } S_{\varepsilon}\right\} .
$$

The set $\mathbf{H}_{\varepsilon}(\Omega)$ is a convex closed subset of $H_{\Gamma}^{2}\left(\Omega_{\varepsilon}^{*}\right)^{3}$.

\footnotetext{
${ }^{3}$ such a $v$ exists since the boundary of $\mathcal{S}$ is $\mathcal{C}^{1,1}$
} 
Observe that since the $a_{i j k l}$ 's are $W^{1, \infty}$ in a neighborhood of $S$ and since the boundary of $\mathcal{S}$ is $\mathcal{C}^{1,1}$, the traces on $\partial \mathcal{S}_{\varepsilon}^{ \pm}$of the stress tensor of the elements in $\mathbf{H}_{\varepsilon}(\Omega)$ belong to $H^{1 / 2}\left(\partial \mathcal{S}_{\varepsilon}^{ \pm}\right)$.

For every $u \in \mathbf{H}_{\varepsilon}(\Omega)$, we denote $\sigma_{\nu_{\varepsilon}}(u) \in H^{1 / 2}\left(S_{\varepsilon}\right)$ the restriction to $S_{\varepsilon}$ of $\left(\sigma^{\varepsilon}(u)_{\mid \partial \mathcal{S}_{\varepsilon}^{+}} \nu_{\varepsilon}\right) \cdot \nu_{\varepsilon}$.

From now on, in the left hand-side of problem (5.2) we add the regularization term

$$
\kappa \varepsilon^{2}\left[\nabla e\left(u_{\varepsilon}\right), \nabla e\left(v-u_{\varepsilon}\right)\right]_{\varepsilon}
$$

where

$$
\forall(u, v) \in H_{\Gamma}^{2}\left(\Omega_{\varepsilon}^{*}\right)^{3} \times H_{\Gamma}^{2}\left(\Omega_{\varepsilon}^{*}\right)^{3}, \quad[\nabla e(u), \nabla e(v)]_{\varepsilon} \doteq \int_{\Omega_{\varepsilon}^{*}} \nabla e(u) \nabla e(v) d x .
$$

We are therefore led to consider the following variational inequality:

$$
\left\{\begin{array}{l}
\text { Find } u_{\varepsilon} \in \mathbf{H}_{\varepsilon}(\Omega) \cap \mathcal{K}_{\varepsilon} \text { such that for every } v \in \mathbf{H}_{\varepsilon}(\Omega) \cap \mathcal{K}_{\varepsilon}, \\
\mathbf{a}^{\varepsilon}\left(u_{\varepsilon}, v-u_{\varepsilon}\right)+\kappa \varepsilon^{2}\left[\nabla e\left(u_{\varepsilon}\right), \nabla e\left(v-u_{\varepsilon}\right)\right]_{\varepsilon}+\left(\mu\left|\sigma_{\nu_{\varepsilon}}\left(u_{\varepsilon}\right)\right|,\left|\left[v_{\tau_{\varepsilon}}\right]_{S_{\varepsilon}}\right|-\left|\left[\left(u_{\varepsilon}\right)_{\tau_{\varepsilon}}\right]_{S_{\varepsilon}}\right|\right)_{\varepsilon} \geq\left(f, v-u_{\varepsilon}\right)
\end{array}\right.
$$

where

$$
(\phi, \psi)_{\varepsilon}=\int_{S_{\varepsilon}} \phi \psi d \sigma_{\varepsilon}, \quad \forall(\phi, \psi) \in L^{2}\left(S_{\varepsilon}\right)^{2}
$$

Lemma 6.1. For every $u$ in $\mathbf{H}_{\varepsilon}(\Omega)$, one has

$$
\sqrt{\varepsilon}\left\|e(u)_{\mid \partial \mathcal{S}_{\varepsilon}^{ \pm} \cap S_{\varepsilon}}\right\|_{H^{1 / 2}\left(S_{\varepsilon}\right)} \leq C_{1} \mathbf{N}_{\varepsilon}(u)
$$

and

$$
\sqrt{\varepsilon}\left\|\sigma_{\nu_{\varepsilon}}(u)\right\|_{H^{1 / 2}\left(S_{\varepsilon}\right)} \leq C_{1}\|\mathbf{a}\|_{W^{1, \infty}(S)} \mathbf{N}_{\varepsilon}(u),
$$

where

$$
\|\mathbf{a}\|_{W^{1, \infty}(S)}=\max _{i, j, k, l=1}^{3}\left\|a_{i j k l}\right\|_{W^{1, \infty}(S)} .
$$

The constants do not depend on $\varepsilon$ and $\kappa$, they only depend on $S$.

Proof. Let $\phi$ be in $H^{1}\left(\mathbf{Y}^{*}\right)$, the trace theorem gives

$$
\|\phi\|_{L^{2}\left(\mathcal{S}^{ \pm} \cap S\right)} \leq C\|\phi\|_{H^{1}(\mathbf{Y})}, \quad\|\phi\|_{H^{1 / 2}\left(\mathcal{S}^{ \pm} \cap S\right)}^{\prime} \leq C\|\nabla \phi\|_{L^{2}(\mathbf{Y})} .
$$

Hence

$$
\forall \Phi \in H^{2}(\mathcal{S})^{3}, \quad\|e(\Phi)\|_{H^{1 / 2}\left(\partial \mathcal{S}^{-}\right)} \leq C_{1}\|e(\Phi)\|_{H^{1}(\mathcal{S})} .
$$

The constants depend only on $S$. Estimate $(6.2)$ follows after $\varepsilon$-scaling applied to the $e_{i j}(u)$ 's. Then $(6.3)$ is an immediate consequence of $(6.2)$.

Corollary 6.2. For every $u$ in $\mathbf{H}_{\varepsilon}(\Omega)$

$$
\left\|\mathcal{T}_{\varepsilon}^{b}\left(\sigma_{\nu_{\varepsilon}}(u)\right)\right\|_{L^{2}\left(\Omega \times S_{\varepsilon}\right)} \leq C \mathbf{N}_{\varepsilon}(u)
$$

The constant does not depend on $\varepsilon$.

\section{$7 \quad$ Existence results for $\varepsilon$ and friction fixed}

As a consequence of Lemma 3.7, one has

$$
|(f, u)| \leq C\|f\|_{L^{2}(\Omega)}\|e(u)\|_{L^{2}\left(\Omega_{\varepsilon}^{*}\right)}, \quad \forall u \in \mathbf{H}_{\varepsilon}(\Omega) .
$$

Now, consider the contact problem associated to (6.1) with constant friction

$$
\left\{\begin{array}{l}
\text { Find } u \in \mathbf{H}_{\varepsilon}(\Omega) \cap \mathcal{K}_{\varepsilon} \text { such that for every } v \in \mathbf{H}_{\varepsilon}(\Omega) \cap \mathcal{K}_{\varepsilon}, \\
\mathbf{a}^{\varepsilon}(u, v-u)+\kappa \varepsilon^{2}[\nabla e(u), \nabla e(v-u)]_{\varepsilon}+\left(G,\left|\left[v_{\tau_{\varepsilon}}\right]_{S_{\varepsilon}}\right|-\left|\left[u_{\tau_{\varepsilon}}\right]_{S_{\varepsilon}}\right|\right)_{\varepsilon} \geq(f, v-u)
\end{array}\right.
$$


where the linear form $G$ is an element of the cone

$$
\mathcal{C}_{\varepsilon}^{* *} \doteq\left\{G \in L^{2}\left(S_{\varepsilon}\right) \mid G \geq 0 \text { a.e. on } S_{\varepsilon}\right\} .
$$

The functional $J_{\varepsilon}$ defined in $\mathbf{H}_{\varepsilon}(\Omega) \cap \mathcal{K}_{\varepsilon}$ by

$$
J_{\varepsilon}: u \in \mathbf{H}_{\varepsilon}(\Omega) \cap \mathcal{K}_{\varepsilon} \longmapsto \frac{1}{2} \mathbf{a}^{\varepsilon}(u, u)+\frac{\kappa \varepsilon^{2}}{2}[\nabla e(u), \nabla e(u)]_{\varepsilon}+\left(G,\left|\left[u_{\tau_{\varepsilon}}\right]_{S_{\varepsilon}}\right|\right)_{\varepsilon}-(f, u)
$$

is strictly convex, weakly lower semicontinuous and due to $3.101,2$, it satisfies

$$
\lim _{u \in \mathbf{H}_{\varepsilon}(\Omega) \cap \mathcal{K}_{\varepsilon}, \mathbf{N}_{\varepsilon}(u) \rightarrow+\infty} J_{\varepsilon}(u)=+\infty .
$$

As a consequence, there exists a unique solution for the corresponding minimization problem or equivalently for the problem (7.1) (see [4]).

\section{Estimates}

In this section, the solution to problem (7.1) is denoted $u_{\varepsilon, G}$.

Theorem 8.1. The solution $u_{\varepsilon, G}$ of the contact problem (7.1) satisfies

$$
\begin{aligned}
& \sqrt{\kappa} \varepsilon\left\|\nabla e\left(u_{\varepsilon, G}\right)\right\|_{L^{2}\left(\Omega_{\varepsilon}^{*}\right)} \leq C\|f\|_{L^{2}(\Omega)}, \\
& \left\|u_{\varepsilon, G}\right\|_{H^{1}\left(\Omega_{\varepsilon}^{*}\right)} \leq C\|f\|_{L^{2}(\Omega)} \\
& \left\|\left[u_{\varepsilon, G}\right]_{S_{\varepsilon}}\right\|_{H^{1 / 2}\left(S_{\varepsilon}\right)} \leq C \sqrt{\varepsilon}\|f\|_{L^{2}(\Omega)} .
\end{aligned}
$$

Furthermore, one has

$$
\sqrt{\varepsilon}\left\|\sigma_{\nu_{\varepsilon}}\left(u_{\varepsilon, G}\right)\right\|_{H^{1 / 2}\left(S_{\varepsilon}\right)} \leq C_{2}\left(1+\frac{1}{\sqrt{\kappa}}\right)\|f\|_{L^{2}(\Omega)} .
$$

Moreover, the solution $u_{\varepsilon, G}$ depends continuously on the given friction $G$ and one has

$$
\left\|\sigma_{\nu_{\varepsilon}}\left(u_{\varepsilon, G_{1}}\right)-\sigma_{\nu_{\varepsilon}}\left(u_{\varepsilon, G_{2}}\right)\right\|_{H^{1 / 2}\left(S_{\varepsilon}\right)} \leq \frac{C_{0} C_{1}\|\mathbf{a}\|_{W^{1, \infty}(S)}}{\min \{\bar{\alpha}, \kappa\}}\left\|G_{1}-G_{2}\right\|_{L^{2}\left(S_{\varepsilon}\right)}, \quad \forall\left(G_{1}, G_{2}\right) \in\left(\mathcal{C}_{\varepsilon}^{* *}\right)^{2} .
$$

The constants do not depend on $\varepsilon$ and $\kappa$.

Proof. From equality (7.1), one obtains

$$
\mathbf{a}^{\varepsilon}\left(u_{\varepsilon, G}, u_{\varepsilon, G}\right)+\kappa \varepsilon^{2}\left[\nabla e\left(u_{\varepsilon, G}\right), \nabla e\left(u_{\varepsilon, G}\right)\right]_{\varepsilon}+\left(G,\left|\left[\left(u_{\varepsilon, G}\right)_{\tau_{\varepsilon}}\right]_{S_{\varepsilon}}\right|\right)_{\varepsilon} \leq\left(f, u_{\varepsilon, G}\right) .
$$

Then estimates (8.1) follow the ones of Proposition 3.7 while (8.2) is a consequence of 6.3 .

Now, problem (7.1) with $G_{1}$ and then $G_{2}$ together with the estimates 3.10$)_{2}$ gives

$$
\begin{aligned}
& \bar{\alpha}\left\|e\left(u_{\varepsilon, G_{1}}\right)-e\left(u_{\varepsilon, G_{2}}\right)\right\|_{L^{2}\left(\Omega_{\varepsilon}^{*}\right)}^{2}+\kappa \varepsilon^{2}\left[\nabla e\left(u_{\varepsilon, G_{1}}-u_{\varepsilon, G_{2}}\right), \nabla e\left(u_{\varepsilon, G_{1}}-u_{\varepsilon, G_{2}}\right)\right]_{\varepsilon} \\
& \quad \leq\left\|G_{1}-G_{2}\right\|_{L^{2}\left(S_{\varepsilon}\right)}\left(\left\|\left[\left(u_{\varepsilon, G_{1}}-u_{\varepsilon, G_{2}}\right)_{\tau_{\varepsilon}}\right]_{S_{\varepsilon}}\right\|_{L^{2}\left(S_{\varepsilon}\right)}\right) \\
& \quad \leq C_{0} \sqrt{\varepsilon}\left\|G_{1}-G_{2}\right\|_{L^{2}\left(S_{\varepsilon}\right)}\left\|e\left(u_{\varepsilon, G_{1}}\right)-e\left(u_{\varepsilon, G_{2}}\right)\right\|_{L^{2}\left(\Omega_{\varepsilon}^{*}\right)} .
\end{aligned}
$$

So

$$
\mathbf{N}\left(u_{\varepsilon, G_{1}}-u_{\varepsilon, G_{2}}\right) \leq \frac{C_{0}}{\min \{\bar{\alpha}, \kappa\}} \sqrt{\varepsilon}\left\|G_{1}-G_{2}\right\|_{L^{2}\left(S_{\varepsilon}\right)}
$$

Then estimate $(6.3)$ yields

$$
\left\|\sigma_{\nu_{\varepsilon}}\left(u_{\varepsilon, G_{1}}\right)-\sigma_{\nu_{\varepsilon}}\left(u_{\varepsilon, G_{2}}\right)\right\|_{H^{1 / 2}\left(S_{\varepsilon}\right)} \leq \frac{C_{1}\|\mathbf{a}\|_{W^{1, \infty}(S)}}{\sqrt{\varepsilon}} \mathbf{N}\left(u_{\varepsilon, G_{1}}-u_{\varepsilon, G_{2}}\right) \leq \frac{C_{0} C_{1}\|\mathbf{a}\|_{W^{1, \infty}(S)}}{\min \{\bar{\alpha}, \kappa\}}\left\|G_{1}-G_{2}\right\|_{L^{2}\left(S_{\varepsilon}\right)} .
$$

Therefore 8.3 follows. 


\subsection{Solution of the Coulomb's problem}

A solution of the contact problem with Coulomb friction is characterized by a fix point of the operator

$$
A: G \in \mathcal{C}_{\varepsilon}^{*} \longmapsto \mu\left|\sigma_{\nu_{\varepsilon}}\left(u_{\varepsilon, G}\right)\right| \in \mathcal{C}_{\varepsilon}^{*} .
$$

The existence of a fix point is proved by the Banach fixed-point theorem.

Proposition 8.2. Assume

$$
\frac{C_{0} C_{1}\|\mathbf{a}\|_{W^{1, \infty}(S)}\|\mu\|_{L^{\infty}(\Omega)}}{\min \{\bar{\alpha}, \kappa\}}<1
$$

then problem (6.1) admits a unique solution. The constant $C_{0} C_{1}$ depends only on $S$.

Proof. From estimate 8.3 one gets

$$
\begin{aligned}
\left\|\mu\left(\sigma_{\nu_{\varepsilon}}\left(u_{\varepsilon, G_{1}}\right)-\sigma_{\nu_{\varepsilon}}\left(u_{\varepsilon, G_{2}}\right)\right)\right\|_{L^{2}\left(S_{\varepsilon}\right)} & \leq\|\mu\|_{L^{\infty}(\Omega)}\left\|\sigma_{\nu_{\varepsilon}}\left(u_{\varepsilon, G_{1}}\right)-\sigma_{\nu_{\varepsilon}}\left(u_{\varepsilon, G_{2}}\right)\right\|_{L^{2}\left(S_{\varepsilon}\right)} \\
& \leq \frac{C_{0} C_{1}\|\mathbf{a}\|_{W^{1, \infty}(S)}\|\mu\|_{L^{\infty}(\Omega)}}{\min \{\bar{\alpha}, \kappa\}}\left\|G_{1}-G_{2}\right\|_{L^{2}\left(S_{\varepsilon}\right)} .
\end{aligned}
$$

Now, consider the map $A$ defined by (8.5). It is continuous and if the condition 8.6$)$ is satisfied then the Banach fixed-point theorem gives a unique solution to the Coulomb friction problem 6.1).

Solutions of (6.1) can also be obtained by Schauder's theorem.

Theorem 8.3 (Schauder's theorem). Let $\mathcal{X}$ be a Banach space and let $\mathcal{Y} \subset \mathcal{X}$ be a convex compact subset. Then every continuous map (for the strong topology of $\mathcal{X}$ ) $A: \mathcal{Y} \rightarrow \mathcal{Y}$ has a fixed point in $\mathcal{Y}$.

Proposition 8.4. For every $\varepsilon$ the problem (6.1) admits solutions. The solutions satisfy

$$
\sqrt{\varepsilon}\left\|\mu \sigma_{\nu_{\varepsilon}}\left(u_{\varepsilon, G}\right)\right\|_{H^{1 / 2}\left(S_{\varepsilon}\right)} \leq C_{1} C_{2}\left(1+\frac{1}{\sqrt{\kappa}}\right)\|\mu\|_{W^{1, \infty}(\Omega)}\|f\|_{L^{2}(\Omega)} .
$$

The constant does not depend on $\varepsilon$.

Proof. Since $\mu$ belongs to $W^{1, \infty}(\Omega)$ and due to $8.1-(8.2)$, one has

$$
\sqrt{\varepsilon}\left\|\mu \sigma_{\nu_{\varepsilon}}\left(u_{\varepsilon, G}\right)\right\|_{H^{1 / 2}\left(S_{\varepsilon}\right)} \leq C_{1} \sqrt{\varepsilon}\|\mu\|_{W^{1, \infty}(\Omega)}\left\|\sigma_{\nu_{\varepsilon}}\left(u_{\varepsilon, G}\right)\right\|_{H^{1 / 2}\left(S_{\varepsilon}\right)} \leq C_{1} C_{2}\left(1+\frac{1}{\sqrt{\kappa}}\right)\|\mu\|_{W^{1, \infty}(\Omega)}\|f\|_{L^{2}(\Omega)} .
$$

Now, set

$$
R=C_{1} C_{2}\left(1+\frac{1}{\sqrt{\kappa}}\right)\|\mu\|_{W^{1, \infty}(\Omega)}\|f\|_{L^{2}(\Omega)}
$$

Therefore, one has

$$
\forall G \in \mathcal{C}_{\varepsilon}^{* *}, \quad \mu \sigma_{\nu_{\varepsilon}}\left(u_{\varepsilon, G}\right) \text { belongs to } \mathcal{C}_{\varepsilon}^{* *} \text { and } \quad\|G\|_{H^{1 / 2}\left(S_{\varepsilon}\right)} \leq \frac{R}{\sqrt{\varepsilon}} \Longrightarrow\left\|\mu \sigma_{\nu_{\varepsilon}}\left(u_{\varepsilon, G}\right)\right\|_{H^{1 / 2}\left(S_{\varepsilon}\right)} \leq \frac{R}{\sqrt{\varepsilon}} .
$$

Applying Schauder's theorem with the map $A$ (see (8.5)), $\mathcal{X}=L^{2}\left(S_{\varepsilon}\right)$ (endowed with the strong topology) and choosing $\mathcal{Y}=\left\{G \in \mathcal{C}_{\varepsilon}^{* *} \quad \mid\|G\|_{H^{1 / 2}\left(S_{\varepsilon}\right)} \leq \frac{R}{\sqrt{\varepsilon}}\right\}$ (which is a compact convex subset of $\mathcal{X}$ ) give solutions to problem 6.1).

\section{Homogenization process}

In this section, we denote $u_{\varepsilon}$ the solution to problem (6.1).

From (8.1) the displacement $u_{\varepsilon}$ satisfies the following estimates:

$$
\begin{aligned}
& \varepsilon\left\|\nabla e\left(u_{\varepsilon}\right)\right\|_{L^{2}\left(\Omega_{\varepsilon}^{*}\right)} \leq C\|f\|_{L^{2}(\Omega)}, \\
& \left\|u_{\varepsilon}\right\|_{H^{1}\left(\Omega_{\varepsilon}^{*}\right)} \leq C\|f\|_{L^{2}(\Omega)}, \\
& \left\|\left[u_{\varepsilon}\right]_{S_{\varepsilon}}\right\|_{H^{1 / 2}\left(S_{\varepsilon}\right)} \leq C \sqrt{\varepsilon}\|f\|_{L^{2}(\Omega)}, \\
& \sqrt{\varepsilon}\left\|\sigma_{\nu_{\varepsilon}}\left(u_{\varepsilon}\right)\right\|_{H^{1 / 2}\left(S_{\varepsilon}\right)} \leq C\|f\|_{L^{2}(\Omega)} .
\end{aligned}
$$

The constants do not depend on $\varepsilon$. 


\subsection{A compactness result}

Below we give a result related to the unfolding method.

Definition 3. Let $\omega$ be an open set strictly included in $\Omega$. For every $\varepsilon \leq \operatorname{dist}(\omega, \partial \Omega) / 4$ and for every $\phi \in L^{p}\left(\Omega_{\varepsilon}^{*}\right)$, $p \in[1,+\infty]$, we define $\mathcal{Q}_{\varepsilon}^{\diamond}(\phi) \in W^{1, \infty}\left(\omega ; L^{p}\left(\mathbf{Y}^{*}\right)\right)$ by $Q_{1}$ interpolation. We set

for $\xi \in \Xi_{\varepsilon}, \quad \mathcal{Q}_{\varepsilon}^{\diamond}(\phi)(\varepsilon \xi, y)=\mathcal{T}_{\varepsilon}^{*}(\phi)(\varepsilon \xi, y) \quad$ for a.e. $y \in \mathbf{Y}^{*}$,

for $x \in \omega, \quad \mathcal{Q}_{\varepsilon}^{\diamond}(\phi)(x, y)$ is the $Q_{1}$ interpolate of $\mathcal{Q}_{\varepsilon}^{\diamond}(\phi)$ at the vertices of the cell $\left(\varepsilon\left[\frac{x}{\varepsilon}\right]_{\varepsilon}+\varepsilon \mathbf{Y}\right) \times\{y\}$,

for a.e. $y \in \mathbf{Y}^{*}$.

Lemma 9.1. Assume $\omega \Subset \Omega$. If $\varepsilon$ is small enough, for every $\phi \in H^{1}\left(\Omega_{\varepsilon}^{*}\right)$ then $\mathcal{Q}_{\varepsilon}^{\diamond}(\phi)$ belongs to $H^{1}\left(\omega ; H^{1}\left(\mathbf{Y}^{*}\right)\right)$ and

$$
\left\|\mathcal{Q}_{\varepsilon}^{\diamond}(\phi)\right\|_{H^{1}\left(\omega ; H^{1}\left(\mathbf{Y}^{*}\right)\right)} \leq C\|\phi\|_{H^{1}\left(\Omega_{\varepsilon}^{*}\right)} .
$$

The constant does not depend on $\varepsilon$ and $\omega$.

Proof. See [7, Propositions 2.6-2.7] for the proof.

Lemma 9.2. Suppose $\omega \Subset \Omega$. One has

$$
\left\|\mathcal{Q}_{\varepsilon}^{\diamond}\left(e\left(u_{\varepsilon}\right)\right)\right\|_{H^{1}\left(\omega ; H^{1}\left(\mathbf{Y}^{*}\right)\right)} \leq C\|f\|_{L^{2}(\Omega)}, \quad\left\|\mathcal{Q}_{\varepsilon}^{\diamond}\left(e\left(u_{\varepsilon}\right)\right)-\mathcal{T}_{\varepsilon}^{*}\left(e\left(u_{\varepsilon}\right)\right)\right\|_{L^{2}\left(\omega ; H^{1}\left(\mathbf{Y}^{*}\right)\right)} \leq C \varepsilon\|f\|_{L^{2}(\Omega)}
$$

The constants do not depend on $\varepsilon$ (it depends on $\omega$ ).

Proof. For every function $\phi$ belonging to $L^{2}\left(\Omega_{\varepsilon}^{*}\right)$ with support strictly included in $\Omega$ and for $\varepsilon$ small enough, set

$$
\Delta_{\mathbf{k}} \phi=\phi(\cdot+\varepsilon \mathbf{k})-\phi, \quad \mathbf{k}=\mathbf{e}_{i}, \quad i \in\{1,2,3\}
$$

$\Delta_{\mathbf{k}} \phi$ also belongs to $L^{2}\left(\Omega_{\varepsilon}^{*}\right)$.

Step 1. An equicontinuity result.

Let $\omega$ be an open set such that $\omega \Subset \Omega^{\prime} \Subset \Omega$ (see the assumption on $\mu$ ) with $\operatorname{dist}(\omega, \partial \Omega)=\delta>0$. There exists a function $\rho_{\omega} \in \mathcal{D}(\Omega)$ such that

$$
\begin{aligned}
& 0 \leq \rho_{\omega}(x) \leq 1, \quad \forall x \in \Omega, \\
& \rho_{\omega}(x)=0 \quad \text { if } \operatorname{dist}(x, \partial \Omega) \leq \frac{\delta}{4}, \quad x \in \Omega, \\
& \rho_{\omega}(x)=1 \quad \text { if } \operatorname{dist}(x, \partial \Omega) \geq \frac{\delta}{2}, \quad x \in \Omega .
\end{aligned}
$$

Function $\rho_{\omega}$ satisfies $\left((i, j) \in\{1,2,3\}^{2}\right)$

$$
\left\|\frac{\partial \rho_{\omega}}{\partial x_{i}}\right\|_{L^{\infty}(\Omega)} \leq \frac{C}{\delta}, \quad\left\|\frac{\partial^{2} \rho_{\omega}}{\partial x_{i} \partial x_{j}}\right\|_{L^{\infty}(\Omega)} \leq \frac{C}{\delta^{2}}, \quad\left\|\Delta_{\mathbf{k}} \frac{\partial \rho_{\omega}}{\partial x_{i}}\right\|_{L^{\infty}(\Omega)} \leq C \frac{\varepsilon}{\delta^{2}}, \quad\left\|\Delta_{\mathbf{k}} \frac{\partial^{2} \rho_{\omega}}{\partial x_{i} \partial x_{j}}\right\|_{L^{\infty}(\Omega)} \leq C \frac{\varepsilon}{\delta^{3}}
$$

where the constant depends on $\partial \Omega$. Below, in the estimates we will not mention the dependence of constants with respect to $\delta$ since the open set $\omega$ is fixed.

Let $v$ be in $\mathcal{K}_{\varepsilon}$. Set $v_{\varepsilon}=u_{\varepsilon}+\rho_{\omega}\left(v-\rho_{\omega} u_{\varepsilon}\right)$. First, observe that $v=u_{\varepsilon}$ in the neighborhood of the boundary of $\Omega$, also note that $v_{\varepsilon}$ is an admissible test displacement (it belongs to $\mathcal{K}_{\varepsilon}$ ). Denote

$$
\mathbf{u}_{\varepsilon}=\rho_{\omega} u_{\varepsilon}, \quad \mathbf{f}=\rho_{\omega} f
$$

In 6.1 choose as test function $v_{\varepsilon}$, rewrite the inequality in terms of $\mathbf{u}_{\varepsilon}$ and $\mathbf{f}$, shifting the terms with derivatives of $\rho_{\omega}$ into the right-hand side and additionally due to the assumption on $\mu$ observe that

$$
\mu \sigma_{\nu_{\varepsilon}}\left(\mathbf{u}_{\varepsilon}\right)=\mu \sigma_{\nu_{\varepsilon}}\left(u_{\varepsilon}\right)
$$


One obtains

$$
\begin{aligned}
& \mathbf{a}^{\varepsilon}\left(\mathbf{u}_{\varepsilon}, v-\mathbf{u}_{\varepsilon}\right)+\kappa \varepsilon^{2}\left[\mathbf{u}_{\varepsilon}, v-\mathbf{u}_{\varepsilon}\right]_{\varepsilon}-\left(\mu \sigma_{\nu_{\varepsilon}}\left(\mathbf{u}_{\varepsilon}\right),\left|\left[v_{\tau_{\varepsilon}}\right]\right|_{S_{\varepsilon}}-\left|\left[\left(\mathbf{u}_{\varepsilon}\right)_{\tau_{\varepsilon}}\right]\right|_{S_{\varepsilon}}\right)_{\varepsilon} \\
& \geq \int_{\Omega_{\varepsilon}^{*}} \mathbf{f}\left(v-\mathbf{u}_{\varepsilon}\right) d x+\mathbf{b}_{\varepsilon}\left(u_{\varepsilon}, v-\mathbf{u}_{\varepsilon}\right) \quad \text { for every } v \in \mathcal{K}_{\varepsilon},
\end{aligned}
$$

where $\mathbf{b}_{\varepsilon}$ is a bilinear form. For every $w \in H_{\Gamma}^{1}\left(\Omega_{\varepsilon}^{*}\right)^{3}$ one has

$$
\mathbf{b}_{\varepsilon}\left(u_{\varepsilon}, w\right)=\mathbf{a}^{\varepsilon}\left(\rho_{\omega} u_{\varepsilon}, w\right)-\mathbf{a}^{\varepsilon}\left(u_{\varepsilon}, \rho_{\omega} w\right)+\kappa \varepsilon^{2}\left(\left[\rho_{\omega} u_{\varepsilon}, w\right]_{\varepsilon}-\left[u_{\varepsilon}, \rho_{\omega} w\right]_{\varepsilon}\right)
$$

which is also equal to

$$
\begin{aligned}
& \mathbf{b}_{\varepsilon}\left(u_{\varepsilon}, w\right)=\int_{\Omega_{\varepsilon}^{*}} a_{i j k l}^{\varepsilon}\left[e_{i j}\left(u_{\varepsilon}\right) \frac{\partial \rho_{\omega}}{\partial x_{l}} w_{k}-\frac{\partial \rho_{\omega}}{\partial x_{j}}\left(u_{\varepsilon}\right)_{i} e_{k l}(w)\right] d x \\
& +\kappa \varepsilon^{2} \int_{\Omega_{\varepsilon}^{*}}\left[\left(\frac{\partial^{2} \rho_{\omega}}{\partial x_{l} \partial x_{i}}\left(u_{\varepsilon}\right)_{j}+2 \frac{\partial \rho_{\omega}}{\partial x_{l}} e_{i j}\left(u_{\varepsilon}\right)\right) \frac{\partial e_{i j}(w)}{\partial x_{l}}-\frac{\partial e_{i j}\left(u_{\varepsilon}\right)}{\partial x_{l}}\left(\frac{\partial^{2} \rho_{\omega}}{\partial x_{l} \partial x_{i}} w_{j}+2 \frac{\partial \rho_{\omega}}{\partial x_{l}} e_{i j}(w)\right)\right] d x,
\end{aligned}
$$

For $\varepsilon$ small enough, the set $\left(\varepsilon \mathbf{k}+\varepsilon S_{\varepsilon}\right) \cap \omega$ is included in $\Omega$ and one has $\mathbf{u}_{\varepsilon}(\cdot \pm \varepsilon \mathbf{k}) \in \mathbf{H}_{\varepsilon}(\Omega) \cap \mathcal{K}_{\varepsilon}$.

Choose $v=\mathbf{u}_{\varepsilon}(\cdot-\varepsilon \mathbf{k}) \in \mathbf{H}_{\varepsilon}(\Omega) \cap \mathcal{K}_{\varepsilon}$ as test displacement in $(9.4)$. Due to the periodicity of the coefficients $a_{i j k l}, \mu$ and after a change of variables, one obtains

$$
\begin{aligned}
& \quad \mathbf{a}^{\varepsilon}\left(\mathbf{u}_{\varepsilon}(\cdot+\varepsilon \mathbf{k}), \mathbf{u}_{\varepsilon}-\mathbf{u}_{\varepsilon}(\cdot+\varepsilon \mathbf{k})\right)+\kappa \varepsilon^{2}\left[\nabla e\left(\mathbf{u}_{\varepsilon}(\cdot+\varepsilon \mathbf{k})\right), \nabla e\left(\mathbf{u}_{\varepsilon}-\mathbf{u}_{\varepsilon}(\cdot+\varepsilon \mathbf{k})\right)\right]_{\varepsilon} \\
& +\left(\mu(\cdot+\varepsilon \mathbf{k})\left|\sigma_{\nu_{\varepsilon}}\left(\mathbf{u}_{\varepsilon}(\cdot+\varepsilon \mathbf{k})\right)\right|,\left|\left[\left(\mathbf{u}_{\varepsilon}\right)_{\tau_{\varepsilon}}\right]_{S_{\varepsilon}}\right|-\left|\left[\left(\mathbf{u}_{\varepsilon}(\cdot+\varepsilon \mathbf{k})\right)_{\tau_{\varepsilon}}\right]_{S_{\varepsilon}}\right|\right)_{\varepsilon} \\
& \quad \geq\left(\mathbf{f}(\cdot+\varepsilon \mathbf{k}), \mathbf{u}_{\varepsilon}-\mathbf{u}_{\varepsilon}(\cdot+\varepsilon \mathbf{k})\right)+\mathbf{b}_{\varepsilon}\left(u_{\varepsilon}(\cdot+\varepsilon \mathbf{k}), \mathbf{u}_{\varepsilon}-\mathbf{u}_{\varepsilon}(\cdot+\varepsilon \mathbf{k})\right) .
\end{aligned}
$$

Besides, since $\mathbf{u}_{\varepsilon}(\cdot+\varepsilon \mathbf{k}) \in \mathbf{H}_{\varepsilon}(\Omega) \cap \mathcal{K}_{\varepsilon}$, from 9.4 one also has

$$
\begin{aligned}
& \mathbf{a}^{\varepsilon}\left(\mathbf{u}_{\varepsilon}, \mathbf{u}_{\varepsilon}(\cdot+\varepsilon \mathbf{k})-\mathbf{u}_{\varepsilon}\right)+\kappa \varepsilon^{2}\left[\nabla e\left(\mathbf{u}_{\varepsilon}\right), \nabla e\left(\mathbf{u}_{\varepsilon}(\cdot+\varepsilon \mathbf{k})-\mathbf{u}_{\varepsilon}\right)\right]_{\varepsilon} \\
+ & \left(\mu \sigma_{\nu_{\varepsilon}}\left(\mathbf{u}_{\varepsilon}\right),\left|\left[\left(\mathbf{u}_{\varepsilon}(\cdot+\varepsilon \mathbf{k})\right)_{\tau_{\varepsilon}}\right]_{S_{\varepsilon}}\right|-\left|\left[\left(\mathbf{u}_{\varepsilon}\right)_{\tau_{\varepsilon}}\right]_{S_{\varepsilon}}\right|\right)_{\varepsilon} \\
& \geq\left(\mathbf{f}, \mathbf{u}_{\varepsilon}(\cdot+\varepsilon \mathbf{k})-\mathbf{u}_{\varepsilon}\right)+\mathbf{b}_{\varepsilon}\left(u_{\varepsilon}, \mathbf{u}_{\varepsilon}(\cdot+\varepsilon \mathbf{k})-\mathbf{u}_{\varepsilon}\right)
\end{aligned}
$$

The above inequalities 9.6-9.7 lead to

$$
\begin{aligned}
& \mathbf{a}^{\varepsilon}\left(\Delta_{\mathbf{k}} \mathbf{u}_{\varepsilon}, \Delta_{\mathbf{k}} \mathbf{u}_{\varepsilon}\right)+\kappa \varepsilon^{2}\left\|\nabla e\left(\Delta_{\mathbf{k}} \mathbf{u}_{\varepsilon}\right)\right\|_{L^{2}\left(\Omega_{\varepsilon}^{*}\right)}^{2}+\left(\mu \sigma_{\nu_{\varepsilon}}\left(\Delta_{\mathbf{k}} \mathbf{u}_{\varepsilon}\right), \Delta_{\mathbf{k}}\left|\left[\left(\mathbf{u}_{\varepsilon}\right)_{\tau_{\varepsilon}}\right]_{S_{\varepsilon}}\right|\right)_{\varepsilon} \\
\leq & \left(\Delta_{\mathbf{k}} \mathbf{f}, \Delta_{\mathbf{k}} \mathbf{u}_{\varepsilon}\right)+\left|\Delta_{\mathbf{k}} \mathbf{b}_{\varepsilon}\right|+\left|\left(\Delta_{\mathbf{k}} \mu\left|\sigma_{\nu_{\varepsilon}}\left(\mathbf{u}_{\varepsilon}\right)(\cdot+\varepsilon \mathbf{k})\right|, \Delta_{\mathbf{k}}\left|\left[\left(\mathbf{u}_{\varepsilon}\right)_{\tau_{\varepsilon}}\right]_{S_{\varepsilon}}\right|\right)_{\varepsilon}\right|
\end{aligned}
$$

where $\Delta_{\mathbf{k}} \mathbf{b}_{\varepsilon}$ is equal to (thanks to (9.5) )

$$
\begin{aligned}
\Delta_{\mathbf{k}} \mathbf{b}_{\varepsilon} \doteq & \int_{\Omega_{\varepsilon}^{*}} a_{i j k l}^{\varepsilon}\left[\Delta_{\mathbf{k}}\left(e_{i j}\left(u_{\varepsilon}\right) \frac{\partial \rho_{\omega}}{\partial x_{l}}\right)\left(\Delta_{\mathbf{k}} \mathbf{u}_{\varepsilon}\right)_{k} d x-\int_{\Omega_{\varepsilon}^{*}} \Delta_{\mathbf{k}}\left(\frac{\partial \rho_{\omega}}{\partial x_{j}}\left(u_{\varepsilon}\right)_{i}\right) e_{k l}\left(\Delta_{\mathbf{k}} \mathbf{u}_{\varepsilon}\right)\right] d x \\
& +\kappa \varepsilon^{2} \int_{\Omega_{\varepsilon}^{*}}\left[\Delta_{\mathbf{k}}\left(\frac{\partial^{2} \rho_{\omega}}{\partial x_{l} \partial x_{i}}\left(u_{\varepsilon}\right)_{j}+2 \frac{\partial \rho_{\omega}}{\partial x_{l}} e_{i j}\left(u_{\varepsilon}\right)\right) \frac{\partial e_{i j}\left(\Delta_{\mathbf{k}} \mathbf{u}_{\varepsilon}\right)}{\partial x_{l}} d x\right. \\
& -\kappa \varepsilon^{2} \int_{\Omega_{\varepsilon}^{*}}\left[\Delta_{\mathbf{k}}\left(\frac{\partial e_{i j}\left(u_{\varepsilon}\right)}{\partial x_{l}} \frac{\partial^{2} \rho_{\omega}}{\partial x_{l} \partial x_{i}}\right) \Delta_{\mathbf{k}}\left(\mathbf{u}_{\varepsilon}\right)_{j}+2 \Delta_{\mathbf{k}}\left(\frac{\partial e_{i j}\left(u_{\varepsilon}\right)}{\partial x_{l}} \frac{\partial \rho_{\omega}}{\partial x_{l}}\right) e_{i j}\left(\Delta_{\mathbf{k}} \mathbf{u}_{\varepsilon}\right)\right] d x .
\end{aligned}
$$

Estimates $(9.1)_{4}$ and 3.7 give

$$
\begin{aligned}
\left|\left(\Delta_{\mathbf{k}} \mu\left|\sigma_{\nu_{\varepsilon}}\left(\mathbf{u}_{\varepsilon}\right)(\cdot+\varepsilon \mathbf{k})\right|, \Delta_{\mathbf{k}}\left|\left[\left(\mathbf{u}_{\varepsilon}\right)_{\tau_{\varepsilon}}\right]_{S_{\varepsilon}}\right|\right)_{\varepsilon}\right| & \leq C \varepsilon\|\nabla \mu\|_{L^{\infty}(\Omega)} \frac{1}{\sqrt{\varepsilon}}\|f\|_{L^{2}(\Omega)}\left\|\Delta_{\mathbf{k}}\left|\left[\left(\mathbf{u}_{\varepsilon}\right)_{\tau_{\varepsilon}}\right]_{S_{\varepsilon}}\right|\right\|_{L^{2}\left(S_{\varepsilon}\right)} \\
& \leq C \varepsilon\|\nabla \mu\|_{L^{\infty}(\Omega)}\|f\|_{L^{2}(\Omega)}\left\|e\left(\Delta_{\mathbf{k}} \mathbf{u}_{\varepsilon}\right) \mid\right\|_{L^{2}\left(\Omega_{\varepsilon}^{*}\right)} .
\end{aligned}
$$

Now, observe that the first and the last integrals in $(9.9)$ are equal to

$$
\begin{aligned}
& \int_{\Omega_{\varepsilon}^{*}} a_{i j k l}^{\varepsilon} \Delta_{\mathbf{k}}\left(e_{i j}\left(u_{\varepsilon}\right) \frac{\partial \rho_{\omega}}{\partial x_{l}}\right)\left(\Delta_{\mathbf{k}} \mathbf{u}_{\varepsilon}\right)_{k} d x=\int_{\Omega_{\varepsilon}^{*}} a_{i j k l}^{\varepsilon} e_{i j}\left(u_{\varepsilon}\right) \frac{\partial \rho_{\omega}}{\partial x_{l}} \Delta_{-\mathbf{k}}\left(\Delta_{\mathbf{k}} \mathbf{u}_{\varepsilon}\right)_{k} d x \\
& \int_{\Omega_{\varepsilon}^{*}}\left[\Delta_{\mathbf{k}}\left(\frac{\partial e_{i j}\left(u_{\varepsilon}\right)}{\partial x_{l}} \frac{\partial^{2} \rho_{\omega}}{\partial x_{l} \partial x_{i}}\right) \Delta_{\mathbf{k}}\left(\mathbf{u}_{\varepsilon}\right)_{j}+2 \Delta_{\mathbf{k}}\left(\frac{\partial e_{i j}\left(u_{\varepsilon}\right)}{\partial x_{l}} \frac{\partial \rho_{\omega}}{\partial x_{l}}\right) e_{i j}\left(\Delta_{\mathbf{k}} \mathbf{u}_{\varepsilon}\right)\right] d x \\
= & \int_{\Omega_{\varepsilon}^{*}}\left[\left(\frac{\partial e_{i j}\left(u_{\varepsilon}\right)}{\partial x_{l}} \frac{\partial^{2} \rho_{\omega}}{\partial x_{l} \partial x_{i}}\right) \Delta_{-\mathbf{k}}\left(\Delta_{\mathbf{k}}\left(\mathbf{u}_{\varepsilon}\right)_{j}\right)+2\left(\frac{\partial e_{i j}\left(u_{\varepsilon}\right)}{\partial x_{l}} \frac{\partial \rho_{\omega}}{\partial x_{l}}\right) \Delta_{-\mathbf{k}}\left(e_{i j}\left(\Delta_{\mathbf{k}} \mathbf{u}_{\varepsilon}\right)\right)\right] d x .
\end{aligned}
$$


Hence, using estimates $(9.3)_{1}$ and $(9.1)$

$$
\begin{aligned}
& \left|\int_{\Omega_{\varepsilon}^{*}} a_{i j k l}^{\varepsilon} \Delta_{\mathbf{k}}\left(e_{i j}\left(u_{\varepsilon}\right) \frac{\partial \rho_{\omega}}{\partial x_{l}}\right)\left(\Delta_{\mathbf{k}} \mathbf{u}_{\varepsilon}\right)_{k} d x\right| \leq C\|f\|_{L^{2}(\Omega)}\|\mathbf{a}\|_{W^{1, \infty}(\Omega)}\left\|\Delta_{-\mathbf{k}}\left(\Delta_{\mathbf{k}} \mathbf{u}_{\varepsilon}\right)\right\|_{L^{2}\left(\Omega_{\varepsilon}^{*}\right)} \\
& \left|\int_{\Omega_{\varepsilon}^{*}}\left[\Delta_{\mathbf{k}}\left(\frac{\partial e_{i j}\left(u_{\varepsilon}\right)}{\partial x_{l}} \frac{\partial^{2} \rho_{\omega}}{\partial x_{l} \partial x_{i}}\right) \Delta_{\mathbf{k}}\left(\mathbf{u}_{\varepsilon}\right)_{j}+2 \Delta_{\mathbf{k}}\left(\frac{\partial e_{i j}\left(u_{\varepsilon}\right)}{\partial x_{l}} \frac{\partial \rho_{\omega}}{\partial x_{l}}\right) e_{i j}\left(\Delta_{\mathbf{k}} \mathbf{u}_{\varepsilon}\right)\right] d x\right| \\
& \left.\leq C\|f\|_{L^{2}(\Omega)}\left(\| \Delta_{-\mathbf{k}}\left(\Delta_{\mathbf{k}}\left(\mathbf{u}_{\varepsilon}\right)\right)\right)\left\|_{L^{2}\left(\Omega_{\varepsilon}^{*}\right)}+\right\| \Delta_{-\mathbf{k}}\left(e_{i j}\left(\Delta_{\mathbf{k}} \mathbf{u}_{\varepsilon}\right)\right) \|_{L^{2}\left(\Omega_{\varepsilon}^{*}\right)}\right) .
\end{aligned}
$$

Therefore, with 9.3$)_{2,3}$ and again estimates (9.1) one obtains

$$
\left\|\Delta_{\mathbf{k}}\left(\frac{\partial \rho_{\omega}}{\partial x_{j}}\left(u_{\varepsilon}\right)_{i}\right)\right\|_{L^{2}\left(\Omega_{\varepsilon}^{*} \cap \omega\right)} \leq C \varepsilon\|f\|_{L^{2}(\Omega)}, \quad\left\|\Delta_{\mathbf{k}}\left(\frac{\partial^{2} \rho_{\omega}}{\partial x_{l} \partial x_{i}}\left(u_{\varepsilon}\right)_{j}+2 \frac{\partial \rho_{\omega}}{\partial x_{l}} e_{i j}\left(u_{\varepsilon}\right)\right)\right\|_{L^{2}\left(\Omega_{\varepsilon}^{*} \cap \omega\right)} \leq C\|f\|_{L^{2}(\Omega)} .
$$

The constants only depend on $\omega$. Besides,

$$
\begin{aligned}
& \left\|\Delta_{-\mathbf{k}}\left(\Delta_{\mathbf{k}}\left(\mathbf{u}_{\varepsilon}\right)\right)\right\|_{L^{2}\left(\Omega_{\varepsilon}^{*}\right)} \leq C \varepsilon\left\|\nabla\left(\Delta_{\mathbf{k}}\left(\mathbf{u}_{\varepsilon}\right)\right)\right\|_{L^{2}\left(\Omega_{\varepsilon}^{*}\right)} \leq C \varepsilon\left\|e\left(\Delta_{\mathbf{k}}\left(\mathbf{u}_{\varepsilon}\right)\right)\right\|_{L^{2}\left(\Omega_{\varepsilon}^{*}\right)}, \\
& \left\|\Delta_{-\mathbf{k}}\left(e\left(\Delta_{\mathbf{k}} \mathbf{u}_{\varepsilon}\right)\right)\right\|_{L^{2}\left(\Omega_{\varepsilon}^{*}\right)}\|\leq C \varepsilon\| \nabla\left(\Delta_{\mathbf{k}} e\left(\mathbf{u}_{\varepsilon}\right)\right)\left\|_{L^{2}\left(\Omega_{\varepsilon}^{*}\right)} \leq C \varepsilon\right\| \nabla\left(e\left(\Delta_{\mathbf{k}} \mathbf{u}_{\varepsilon}\right)\right) \|_{L^{2}\left(\Omega_{\varepsilon}^{*}\right)}
\end{aligned}
$$

Summarizing the above equalities and estimates lead to

$$
\left|\Delta_{\mathbf{k}} \mathbf{b}_{\varepsilon}\right| \leq C \varepsilon\|f\|_{L^{2}(\Omega)}\left(\left\|e\left(\Delta_{\mathbf{k}} \mathbf{u}_{\varepsilon}\right)\right\|_{L^{2}\left(\Omega_{\varepsilon}^{*}\right)}+\varepsilon\left\|\nabla\left(e\left(\Delta_{\mathbf{k}} \mathbf{u}_{\varepsilon}\right)\right)\right\|_{L^{2}\left(\Omega_{\varepsilon}^{*}\right)}\right) .
$$

Hence, (9.8) together with 9.10-9.11) yield

$$
\begin{aligned}
& \bar{\alpha}\left\|e\left(\Delta_{\mathbf{k}} u_{\varepsilon}\right)\right\|_{L^{2}\left(\Omega_{\varepsilon}^{*}\right)}^{2}+\kappa \varepsilon^{2}\left\|\nabla e\left(\Delta_{\mathbf{k}} u_{\varepsilon}\right)\right\|_{L^{2}\left(\Omega_{\varepsilon}^{*}\right)}^{2} \\
\leq & \varepsilon\|\mu\|_{L^{\infty}(\Omega)}\left\|\sigma_{\nu_{\varepsilon}}\left(\Delta_{\mathbf{k}} u_{\varepsilon}\right)\right\|_{L^{2}\left(S_{\varepsilon}\right)}\left\|\Delta_{\mathbf{k}}\left[\left(u_{\varepsilon}\right)_{\tau_{\varepsilon}}\right]\right\|_{L^{2}\left(S_{\varepsilon}\right)} \\
+ & C\|f\|_{L^{2}(\Omega)}\left\|\Delta_{-\mathbf{k}}\left(\Delta_{\mathbf{k}} u_{\varepsilon}\right)\right\|_{L^{2}\left(\Omega_{\varepsilon}^{*}\right)}+C \varepsilon\|f\|_{L^{2}(\Omega)} \mathbf{N}_{\varepsilon}\left(\Delta_{\mathbf{k}} u_{\varepsilon}\right) .
\end{aligned}
$$

Using (3.7), (6.3) that gives

$$
\begin{aligned}
& \bar{\alpha}\left\|e\left(\Delta_{\mathbf{k}} u_{\varepsilon}\right)\right\|_{L^{2}\left(\Omega_{\varepsilon}^{*}\right)}^{2}+\kappa \varepsilon^{2}\left\|\nabla e\left(\Delta_{\mathbf{k}} u_{\varepsilon}\right)\right\|_{L^{2}\left(\Omega_{\varepsilon}^{*}\right)}^{2} \\
\leq & \|\mu\|_{L^{\infty}(\Omega)} \frac{C_{1}}{\sqrt{\varepsilon}}\|\mathbf{a}\|_{W^{1, \infty}(S)} \mathbf{N}_{\varepsilon}\left(\Delta_{\mathbf{k}} u_{\varepsilon}\right) C_{0} \sqrt{\varepsilon}\left\|e\left(\Delta_{\mathbf{k}} u_{\varepsilon}\right)\right\|_{L^{2}\left(\Omega_{\varepsilon}^{*}\right)}+C \varepsilon\|f\|_{L^{2}(\Omega)} \mathbf{N}_{\varepsilon}\left(\Delta_{\mathbf{k}} u_{\varepsilon}\right) .
\end{aligned}
$$

Thus

$$
\min \{\bar{\alpha}, \kappa\} \mathbf{N}_{\varepsilon}^{2}\left(\Delta_{\mathbf{k}} u_{\varepsilon}\right) \leq C_{0} C_{1}\|\mu\|_{L^{\infty}(\Omega)}\|\mathbf{a}\|_{W^{1, \infty}(S)} \mathbf{N}_{\varepsilon}^{2}\left(\Delta_{\mathbf{k}} u_{\varepsilon}\right)+C \varepsilon\|f\|_{L^{2}(\Omega)} \mathbf{N}_{\varepsilon}\left(\Delta_{\mathbf{k}} u_{\varepsilon}\right)
$$

At this point one deduces that under assumption (8.6), estimate

$$
\mathbf{N}_{\varepsilon}\left(\Delta_{\mathbf{k}} u_{\varepsilon}\right) \leq C \varepsilon\|f\|_{L^{2}(\Omega)}, \quad \mathbf{k}=\mathbf{e}_{i}, \quad i \in\{1,2,3\}
$$

holds true. The constant does not depend on $\varepsilon$ (it depends on $\omega$ ).

Step 2. We prove the estimates of the lemma.

First the above estimate 9.12 and 9.1$)_{1}$ lead to

$$
\begin{aligned}
& \left\|\mathcal{T}_{\varepsilon}^{*}\left(e\left(u_{\varepsilon}\right)\right)\right\|_{L^{2}\left(\Omega ; H^{1}\left(\mathbf{Y}^{*}\right)\right)} \leq C\|f\|_{L^{2}(\Omega)}, \\
& \sum_{i=1}^{3}\left\|\mathcal{T}_{\varepsilon}^{*}\left(e\left(u_{\varepsilon}\right)\left(\cdot+\varepsilon \mathbf{e}_{i}, \cdot\right)\right)-\mathcal{T}_{\varepsilon}^{*}\left(e\left(u_{\varepsilon}\right)\right)\right\|_{L^{2}\left(\omega ; H^{1}\left(\mathbf{Y}^{*}\right)\right)} \leq C \varepsilon\|f\|_{L^{2}(\Omega)}
\end{aligned}
$$

which in turn yield $(9.2)$ (see [5]).

As a consequence of the above lemma one has

$$
\left\|\mathcal{Q}_{\varepsilon}^{\diamond}\left(e\left(u_{\varepsilon}\right)\right)-\mathcal{T}_{\varepsilon}^{*}\left(e\left(u_{\varepsilon}\right)\right)\right\|_{L^{2}\left(\omega ; H^{1 / 2}\left(\partial \mathcal{S}^{ \pm}\right)\right)} \leq C \varepsilon\|f\|_{L^{2}(\Omega)} .
$$

The constants do not depend on $\varepsilon$ (it depends on $\omega$ ). 
Lemma 9.3. There exists a constant $C^{*}$ which only depends on $\mathbf{Y}^{*}$ such that for every $(v, \widehat{v}) \in H^{1}(\Omega)^{3} \times$ $L^{2}\left(\Omega ; H_{p e r}^{1}\left(\mathbf{Y}^{*}\right)^{3} \cap W\left(\mathbf{Y}^{*}\right)\right)$

$$
C^{*}\left(\|e(v)\|_{L^{2}(\Omega)}+\left\|e_{y}(\widehat{v})\right\|_{L^{2}\left(\Omega \times \mathbf{Y}^{*}\right)}\right) \leq\left\|e(v)+e_{y}(\widehat{v})\right\|_{L^{2}\left(\Omega \times \mathbf{Y}^{*}\right)} .
$$

Proof. Let $\zeta$ be a $3 \times 3$ symmetric matrix and $\widehat{w} \in H_{\text {per }}^{1}\left(\mathbf{Y}^{*}\right)^{3} \cap W\left(\mathbf{Y}^{*}\right)$, one first proves

$$
c^{*}\left(|\zeta|+\left\|e_{y}(\widehat{w})\right\|_{L^{2}\left(\mathbf{Y}^{*}\right)}\right) \leq\left\|\zeta+e_{y}(\widehat{w})\right\|_{L^{2}\left(\mathbf{Y}^{*}\right)} \leq|\zeta|+\left\|e_{y}(\widehat{w})\right\|_{L^{2}\left(\mathbf{Y}^{*}\right)}
$$

The right hand-side inequality is obvious.

To prove the left hand-side, apply the Korn inequality. That gives a rigid displacement $r(y)=\mathbf{a} \wedge y+\mathbf{b}$, $\mathbf{a}, \mathbf{b} \in \mathbb{R}^{3}$ such that

$$
\|\zeta \cdot+r+\widehat{w}\|_{H^{1}\left(\mathbf{Y}^{*}\right)} \leq C\left\|\zeta+e_{y}(\widehat{w})\right\|_{L^{2}\left(\mathbf{Y}^{*}\right)} .
$$

Comparing the traces of the displacement $y \longmapsto \zeta y+r(y)+\widehat{w}(y)$ on the opposite faces of $\mathbf{Y}$ yield

$$
|\zeta| \leq C^{\prime}\|\zeta \cdot+r+\widehat{w}\|_{H^{1}\left(\mathbf{Y}^{*}\right)}
$$

and then $\left\|e_{y}(\widehat{w})\right\|_{L^{2}\left(\mathbf{Y}^{*}\right)} \leq C^{\prime \prime}\left(|\zeta|+\left\|e_{y}(\widehat{w})\right\|_{L^{2}\left(\mathbf{Y}^{*}\right)}\right)$. The constants only depend on $\mathbf{Y}^{*}$. That proves the inequality in the left hand-side of (9.14).

The estimate of lemma is an immediate consequence of (9.14).

Corollary 9.4. Let $(v, \widehat{v})$ be in $H^{1}(\Omega)^{3} \times L^{2}\left(\Omega ; H_{\text {per }}^{1}\left(\mathbf{Y}^{*}\right)\right)^{3}$ satisfying $e(v)+e_{y}(\widehat{v}) \in L^{2}\left(\Omega \times \mathbf{Y}^{*}\right)^{3 \times 3}$. Then $v$ belongs to $H^{2}(\Omega)^{3}$ and $\widehat{v} \in L^{2}\left(\Omega ; H_{\text {per }}^{2}\left(\mathbf{Y}^{*}\right)\right)^{3}$.

\subsection{The unfolded limit variational problem}

The solution of problem (7.1) satisfies the estimates of Theorem 8.1. Set (recall Definition 1)

$$
\begin{aligned}
& \mathcal{K}_{p e r}^{k}\left(\mathbf{Y}^{*}\right) \doteq\left\{\phi \in H_{p e r}^{k}\left(\mathbf{Y}^{*}\right)^{3} \cap W\left(\mathbf{Y}^{*}\right) \mid\left[\phi_{\nu}\right]_{S} \leq 0\right\}, \quad k \in\{1,2\}, \\
& L^{2}\left(\Omega ; \mathcal{K}_{p e r}^{k}\left(\mathbf{Y}^{*}\right)\right) \doteq\left\{\widehat{v} \in L^{2}\left(\Omega ; H_{p e r}^{k}\left(\mathbf{Y}^{*}\right)\right)^{3} \mid \widehat{v}(x, \cdot) \in \mathcal{K}_{p e r}^{k}\left(\mathbf{Y}^{*}\right) \text { for a.e. } x \in \Omega\right\} .
\end{aligned}
$$

Proposition 9.5. There exist a subsequence of $\{\varepsilon\}$, still denoted $\{\varepsilon\}, u$ in $H_{\Gamma}^{1}(\Omega)^{3}$ and $\widehat{u}$ belonging to $L^{2}\left(\Omega ; \mathcal{K}_{\text {per }}^{2}\left(\mathbf{Y}^{*}\right)\right)$ such that

$$
\begin{aligned}
& \mathcal{T}_{\varepsilon}^{*}\left(u_{\varepsilon}\right) \longrightarrow u \quad \text { strongly in } L^{2}\left(\Omega ; H^{1}\left(\mathbf{Y}^{*}\right)\right)^{3}, \\
& \mathcal{T}_{\varepsilon}^{*}\left(\nabla u_{\varepsilon}\right) \rightarrow \nabla u+\nabla_{y} \widehat{u} \quad \text { weakly in } L^{2}\left(\Omega \times \mathbf{Y}^{*}\right)^{3 \times 3}, \\
& \mathcal{T}_{\varepsilon}^{*}\left(e\left(u_{\varepsilon}\right)\right) \longrightarrow e(u)+e_{y}(\widehat{u}) \quad \text { strongly in } L^{2}\left(\Omega^{\prime} \times \mathbf{Y}^{*}\right)^{3 \times 3}, \\
& \mathcal{T}_{\varepsilon}^{*}\left(\nabla e\left(u_{\varepsilon}\right)\right) \rightarrow \nabla_{y} e_{y}(\widehat{u}) \quad \text { weakly in } L^{2}\left(\Omega \times \mathbf{Y}^{*}\right)^{27}, \\
& \frac{1}{\varepsilon} \mathcal{T}_{\varepsilon}^{b}\left(\left[u_{\varepsilon}\right]_{S_{\varepsilon}}\right) \rightarrow[\widehat{u}]_{S} \quad \text { weakly in } L^{2}\left(\Omega ; H^{1 / 2}(S)\right)^{3}, \\
& \mathcal{T}_{\varepsilon}^{b}\left(\sigma_{\nu_{\varepsilon}}\left(u_{\varepsilon}\right)\right) \longrightarrow \Sigma_{\nu} \quad \text { strongly in } L^{2}\left(\Omega^{\prime} \times S\right) .
\end{aligned}
$$

Moreover

$$
\Sigma_{\nu}=\left(\sigma(u)+\sigma_{y}(\widehat{u})_{\mid S}\right) \nu \cdot \nu=\sigma_{\nu}(u)+\sigma_{y, \nu}(\widehat{u})_{\mid S} \leq 0 \quad \text { a.e. in } \quad \Omega \times S
$$

where $\sigma(u)(x, y)=a_{i j k l}(y) e_{i j}(u)(x), \sigma_{y}(\widehat{u})(x, y)=a_{i j k l}(y) e_{i j, y}(\widehat{u})(x, y)$ for a.e. $(x, y) \in \omega \times \mathbf{Y}^{*}$.

Proof. Applying [7, Theorem 2.13] to the sequence $\left\{u_{\varepsilon}\right\}_{\varepsilon}$ gives a subsequence of $\{\varepsilon\}, u$ in $H_{\Gamma}^{1}(\Omega)^{3}$ and $\widehat{u}$ in $L^{2}\left(\Omega ; H_{p e r}^{2}\left(\mathbf{Y}^{*}\right)\right)^{3}$ such that convergences $\left(9.16{ }_{1,2,4}\right.$ hold.

Now, for every open set $\omega$ with Lipschitz boundary satisfying $\omega \Subset \Omega^{\prime} \Subset \Omega$, due to estimates 9.2 and the compact embedding theorems, one obtains

$$
\begin{aligned}
& \mathcal{Q}_{\varepsilon}^{\diamond}\left(e\left(u_{\varepsilon}\right)\right) \rightarrow e(u)+e_{y}(\widehat{u}) \quad \text { weakly in } H^{1}\left(\omega ; H^{1}\left(Y^{*}\right)\right)^{3 \times 3}, \\
& \mathcal{Q}_{\varepsilon}^{\diamond}\left(e\left(u_{\varepsilon}\right)\right) \longrightarrow e(u)+e_{y}(\widehat{u}) \quad \text { strongly in } L^{2}\left(\omega \times Y^{*}\right)^{3 \times 3}, \\
& \mathcal{T}_{\varepsilon}^{*}\left(e\left(u_{\varepsilon}\right)\right) \longrightarrow e(u)+e_{y}(\widehat{u}) \quad \text { strongly in } L^{2}\left(\omega \times Y^{*}\right)^{3 \times 3} .
\end{aligned}
$$


Thus, convergence $(9.16)_{3}$ holds. Moreover, one has

$$
\begin{aligned}
& \mathcal{Q}_{\varepsilon}^{\diamond}\left(e\left(u_{\varepsilon}\right)\right)_{\mid \mathcal{S}^{ \pm}} \rightarrow e(u)+e_{y}(\widehat{u}) \quad \text { weakly in } H^{1}\left(\omega ; H^{1 / 2}\left(\partial \mathcal{S}^{ \pm}\right)\right)^{3 \times 3}, \\
& \mathcal{Q}_{\varepsilon}^{\diamond}\left(e\left(u_{\varepsilon}\right)\right)_{\mathcal{S}^{ \pm}} \longrightarrow e(u)+e_{y}(\widehat{u}) \text { strongly in } L^{2}\left(\omega \times \partial \mathcal{S}^{ \pm}\right)^{3 \times 3} .
\end{aligned}
$$

Estimate 9.13 implies

$$
\mathcal{T}_{\varepsilon}^{b}\left(e\left(u_{\varepsilon}\right)\right)_{\mid \mathcal{S}^{ \pm}} \longrightarrow e(u)+e_{y}(\widehat{u})_{\mid \mathcal{S}^{ \pm}} \quad \text { strongly in } L^{2}\left(\omega \times \mathcal{S}^{ \pm}\right)^{3 \times 3} .
$$

Then, from the above strong convergence, 9.16$)_{6}$ follows.

Set $\widetilde{u}_{\varepsilon}=u_{\varepsilon}-r_{u_{\varepsilon}}$ where $r_{u_{\varepsilon}}$ is defined in Subsection 3.6. From estimates (3.9) in Lemma 3.6 one has

$$
\left\|\mathcal{T}_{\varepsilon}^{*}\left(\widetilde{u}_{\varepsilon}\right)\right\|_{L^{2}\left(\Omega ; H^{1}\left(\mathbf{Y}^{*}\right)\right)} \leq C \varepsilon\|f\|_{L^{2}(\Omega)} .
$$

Then, up to a subsequence, there exists $\widetilde{u} \in L^{2}\left(\Omega ; H^{1}\left(\mathbf{Y}^{*}\right)\right)^{3}$ such that

$$
\frac{1}{\varepsilon} \mathcal{T}_{\varepsilon}^{*}\left(\widetilde{u}_{\varepsilon}\right) \rightarrow \widetilde{u} \quad \text { weakly in } L^{2}\left(\Omega ; H^{1}\left(\mathbf{Y}^{*}\right)\right)^{3}
$$

Hence

$$
\begin{aligned}
& \frac{1}{\varepsilon} \mathcal{T}_{\varepsilon}^{b}\left(\left[\widetilde{u}_{\varepsilon}\right]_{S_{\varepsilon}}\right) \rightarrow[\widetilde{u}]_{S} \quad \text { weakly in } L^{2}\left(\Omega ; H^{1 / 2}(S)\right)^{3}, \\
& \frac{1}{\varepsilon} e_{y}\left(\mathcal{T}_{\varepsilon}^{*}\left(\widetilde{u}_{\varepsilon}\right)\right) \rightarrow e_{y}(\widetilde{u}) \quad \text { weakly in } L^{2}\left(\Omega ; H^{1}\left(\mathbf{Y}^{*}\right)\right)^{3 \times 3}
\end{aligned}
$$

Besides, since $e\left(\widetilde{u}_{\varepsilon}\right)=e\left(u_{\varepsilon}\right)$ in every cell $\varepsilon\left(\xi+\mathbf{Y}^{*}\right), \xi \in \Xi_{\varepsilon}$, that gives $\frac{1}{\varepsilon} e_{y}\left(\mathcal{T}_{\varepsilon}^{*}\left(\widetilde{u}_{\varepsilon}\right)\right)=\mathcal{T}_{\varepsilon}^{*}\left(e\left(\widetilde{u}_{\varepsilon}\right)\right)$. Passing to the limit gives $e_{y}(\widetilde{u})=e(u)+e_{y}(\widehat{u})$ and then there exists $c \in L^{2}(\Omega)^{3}$ such that

$$
\widetilde{u}(x, y)=c(x)+\nabla u(x) y+\widehat{u}(x, y) \quad \text { a.e. in } \Omega \times \mathbf{Y}^{*} .
$$

Finally, since $\left[\widetilde{u}_{\varepsilon}\right]_{S_{\varepsilon}}=\left[u_{\varepsilon}\right]_{S_{\varepsilon}}$ convergence $(9.16)_{5}$ is proved.

Remark 9.6. As a consequence of the above proposition, for every open set $\omega \Subset \Omega$, one has $e(u)+e_{y}(\widehat{u})$ belongs to $H^{1}\left(\omega ; H_{\text {per }}^{1}\left(\mathbf{Y}^{*}\right)\right)^{3 \times 3}$, which implies for $i \in\{1,2,3\}$

$$
e\left(\frac{\partial u}{\partial x_{i}}\right)+e_{y}\left(\frac{\partial \widehat{u}}{\partial x_{i}}\right) \in L^{2}\left(\omega ; H_{p e r}^{1}\left(\mathbf{Y}^{*}\right)\right)^{3 \times 3}
$$

Then Corollary 9.4 yields

$$
u \in H_{\Gamma}^{1}(\Omega)^{3} \cap H_{l o c}^{2}(\Omega)^{3}, \quad \widehat{u} \in H_{l o c}^{1}\left(\Omega ; H_{p e r}^{2}\left(\mathbf{Y}^{*}\right)\right)^{3} .
$$

Theorem 9.7. The pair $(u, \widehat{u}) \in H_{\Gamma}^{1}(\Omega)^{3} \times L^{2}\left(\Omega ; \mathcal{K}_{p e r}^{2}\left(\mathbf{Y}^{*}\right)\right)$ is a solution of the following variational inequality problem:

$$
\begin{aligned}
& \int_{\Omega \times \mathbf{Y}^{*}} a_{i j k l}\left(e_{i j}(u)+e_{y, i j}(\widehat{u})\right)\left(e_{k l}(\Phi-u)+e_{y, k l}(\widehat{\phi}-\widehat{u})\right) d x d y+\kappa \int_{\Omega \times \mathbf{Y}^{*}} \nabla_{y} e_{y}(\widehat{u}) \nabla_{y} e_{y}(\widehat{\phi}-\widehat{u}) d x d y \\
& \quad+\int_{\Omega \times S} \mu\left|\Sigma_{\nu}\right|\left(\left|[\widehat{\phi}]_{\tau}\right|-\left|[\widehat{u}]_{\tau}\right|\right) d x d \sigma_{y} \geq \int_{\Omega} f \cdot(\Phi-u) d x, \quad \forall(\Phi, \widehat{\phi}) \in H_{\Gamma}^{1}(\Omega)^{3} \times L^{2}\left(\Omega ; \mathcal{K}_{p e r}^{2}\left(\mathbf{Y}^{*}\right)\right) .
\end{aligned}
$$

Proof. Consider the test function $v_{\varepsilon}(x)=\Phi(x)+\varepsilon \widehat{\phi}\left(x, \frac{x}{\varepsilon}\right)$, for all $x \in \Omega_{\varepsilon}^{*}$, where $\Phi \in H_{\Gamma}^{1}(\Omega) \cap \mathcal{C}^{\infty}(\bar{\Omega})$ and $\widehat{\phi} \in L^{2}\left(\Omega ; \mathcal{K}_{\text {per }}^{2}\left(\mathbf{Y}^{*}\right)\right) \cap \mathcal{D}\left(\Omega ; H_{\text {per }}^{2}\left(\mathbf{Y}^{*}\right)\right)$. The test displacement $v_{\varepsilon}$ belongs to $\mathcal{K}_{\varepsilon}$. The following strong convergences hold:

$\mathcal{T}_{\varepsilon}^{*}\left(v_{\varepsilon}\right) \longrightarrow \Phi$ strongly in $L^{2}\left(\Omega ; H^{1}\left(\mathbf{Y}^{*}\right)\right)^{3}$, $\mathcal{T}_{\varepsilon}^{*}\left(e\left(v_{\varepsilon}\right)\right) \longrightarrow e(\Phi)+e_{y}(\widehat{\phi}) \quad$ strongly in $L^{2}\left(\Omega \times \mathbf{Y}^{*}\right)^{3 \times 3}$, $\varepsilon \mathcal{T}_{\varepsilon}^{*}\left(\nabla e\left(v_{\varepsilon}\right)\right) \longrightarrow \nabla_{y} e_{y}(\widehat{\phi})$ strongly in $L^{2}\left(\Omega \times \mathbf{Y}^{*}\right)^{27}$, $\frac{1}{\varepsilon} \mathcal{T}_{\varepsilon}^{b}\left(\left[v_{\varepsilon}\right]_{S_{\varepsilon}}\right) \longrightarrow[\widehat{\phi}]_{S} \quad$ strongly in $L^{2}\left(\Omega ; H^{1 / 2}(S)\right)^{3}$, $\mathcal{T}_{\varepsilon}^{b}\left(\sigma_{\nu}\left(v_{\varepsilon}\right)\right) \longrightarrow \sigma_{\nu}(\Phi)+\sigma_{\nu, y}(\widehat{\phi}) \quad$ strongly in $L^{2}(\Omega \times S)$. 
Problem 6.1 also reads

$$
\begin{aligned}
& \mathbf{a}^{\varepsilon}\left(u_{\varepsilon}, u_{\varepsilon}\right)+\kappa \varepsilon^{2}\left[\nabla e\left(u_{\varepsilon}\right), \nabla e\left(u_{\varepsilon}\right)\right]_{\varepsilon}+\left(\mu\left|\sigma_{\nu_{\varepsilon}}\left(u_{\varepsilon}\right)\right|,\left|\left[\left(u_{\varepsilon}\right)_{\tau_{\varepsilon}}\right]_{S_{\varepsilon}}\right|\right)_{\varepsilon}-\left(f, u_{\varepsilon}\right) \\
\leq & \mathbf{a}^{\varepsilon}\left(u_{\varepsilon}, v_{\varepsilon}\right)+\kappa \varepsilon^{2}\left[\nabla e\left(u_{\varepsilon}\right), \nabla e\left(v_{\varepsilon}\right)\right]_{\varepsilon}+\left(\mu\left|\sigma_{\nu_{\varepsilon}}\left(u_{\varepsilon}\right)\right|,\left|\left[\left(v_{\varepsilon}\right)_{\tau_{\varepsilon}}\right]_{S_{\varepsilon}}\right|\right)_{\varepsilon}-\left(f, v_{\varepsilon}\right)
\end{aligned}
$$

Using the properties of the unfolding operators and convergences $\left.(9.16)_{2,6}-9.19\right)_{2,5}$, we obtain

$$
\begin{aligned}
& \lim _{\varepsilon \rightarrow 0} \mathbf{a}^{\varepsilon}\left(u_{\varepsilon}, v_{\varepsilon}\right)=\int_{\Omega \times Y^{*}} a_{i j k l}\left(e_{i j}(u)+e_{y, i j}(\widehat{u})\right)\left(e_{k l}(\Phi)+e_{y, k l}(\widehat{\phi})\right) d x d y, \\
& \lim _{\varepsilon \rightarrow 0} \int_{S_{\varepsilon}} \mu\left|\sigma_{\nu_{\varepsilon}}\left(u_{\varepsilon}\right)\right|\left|\left[\left(v_{\varepsilon}\right)_{\tau_{\varepsilon}}\right]_{S_{\varepsilon}}\right| d \sigma_{\varepsilon}=\int_{\Omega \times S} \mu\left|\Sigma_{\nu}\right|\left|[\widehat{\phi}]_{\tau}\right| d x d \sigma_{y} .
\end{aligned}
$$

Further, due to the lower semi-continuity with respect to weak topology and convergences $[9.16]_{3,4}$ for the unfolded sequences, we obtain

$$
\begin{aligned}
\liminf _{\varepsilon \rightarrow 0}\left(\mathbf{a}^{\varepsilon}\left(u_{\varepsilon}, u_{\varepsilon}\right)+\kappa \varepsilon^{2}[\nabla e\right. & \left.\left.\left(u_{\varepsilon}\right), \nabla e\left(u_{\varepsilon}\right)\right]_{\varepsilon}\right) \\
& \geq \int_{\Omega \times \mathbf{Y}^{*}} a_{i j k l}\left(e_{i j}(u)+e_{y, i j}(\widehat{u})\right)\left(e_{k l}(u)+e_{y, k l}(\widehat{u})\right) d x d y+\kappa\left\|\nabla_{y} e_{y}(\widehat{u})\right\|_{L^{2}\left(\Omega \times \mathbf{Y}^{*}\right)}^{2}
\end{aligned}
$$

while 9.161 yields $\lim _{\varepsilon \rightarrow 0}\left(f, u_{\varepsilon}\right)=\int_{\Omega} f \cdot u d x$.

One has

$$
\begin{aligned}
\int_{S_{\varepsilon}} \mu\left|\sigma_{\nu_{\varepsilon}}\left(u_{\varepsilon}\right)\right|\left|\left[\left(u_{\varepsilon}\right)_{\tau_{\varepsilon}}\right]_{S_{\varepsilon}}\right| d \sigma_{\varepsilon}=\varepsilon \int_{S_{\varepsilon}} \mu \mathbf{1}_{\Omega^{\prime}}\left|\sigma_{\nu_{\varepsilon}}\left(u_{\varepsilon}\right)\right| & \frac{1}{\varepsilon}\left|\left[\left(u_{\varepsilon}\right)_{\tau_{\varepsilon}}\right]_{S_{\varepsilon}}\right| d \sigma_{\varepsilon} \\
& =\int_{\Omega \times S} \mathcal{T}_{\varepsilon}^{b}(\mu) \mathcal{T}_{\varepsilon}^{b}\left(\mathbf{1}_{\Omega^{\prime}}\right)\left|\mathcal{T}_{\varepsilon}^{b}\left(\sigma_{\nu_{\varepsilon}}\left(u_{\varepsilon}\right)\right)\right| \frac{1}{\varepsilon}\left|\mathcal{T}_{\varepsilon}^{b}\left(\left[\left(u_{\varepsilon}\right)_{\tau_{\varepsilon}}\right]_{S_{\varepsilon}}\right)\right| d x d \sigma_{y}
\end{aligned}
$$

From convergences $9.16{ }_{5,6}$, one obtains

$$
\int_{\Omega \times S} \mu \mathbf{1}_{\Omega^{\prime}}\left|\Sigma_{\nu}\right|\left|[\widehat{u}]_{\tau}\right| d x d \sigma_{y}=\lim _{\varepsilon \rightarrow 0} \int_{\Omega \times S} \mathcal{T}_{\varepsilon}^{b}(\mu) \mathcal{T}_{\varepsilon}^{b}\left(\mathbf{1}_{\Omega^{\prime}}\right)\left|\mathcal{T}_{\varepsilon}^{b}\left(\sigma_{\nu_{\varepsilon}}\left(u_{\varepsilon}\right)\right)\right| \frac{1}{\varepsilon}\left|\mathcal{T}_{\varepsilon}^{b}\left(\left[\left(u_{\varepsilon}\right)_{\tau_{\varepsilon}}\right]_{S_{\varepsilon}}\right)\right| d x d \sigma_{y}
$$

Summarizing the above convergences, that leads to

$$
\begin{aligned}
& \int_{\Omega \times \mathbf{Y}^{*}} a_{i j k l}\left(e_{i j}(u)+e_{y, i j}(\widehat{u})\right)\left(e_{k l}(u)+e_{y, k l}(\widehat{u})\right) d x d y+\kappa \int_{\Omega \times \mathbf{Y}^{*}}\left|\nabla_{y} e_{y}(\widehat{u})\right|^{2} d x d y \\
& \quad+\int_{\Omega \times S} \mu\left|\Sigma_{\nu}\right|\left|[\widehat{u}]_{\tau}\right| d x d \sigma_{y}-\int_{\Omega} f \cdot u d x \\
& \leq \int_{\Omega \times \mathbf{Y}^{*}} a_{i j k l}\left(e_{i j}(u)+e_{y, i j}(\widehat{u})\right)\left(e_{k l}(\Phi)+e_{y, k l}(\widehat{\phi})\right) d x d y+\kappa \int_{\Omega \times \mathbf{Y}^{*}} \nabla_{y} e_{y}(\widehat{u}) \nabla_{y} e_{y}(\widehat{\phi}) d x d y \\
& \quad+\int_{\Omega \times S} \mu\left|\Sigma_{\nu}\right|\left|[\widehat{\phi}]_{\tau}\right| d x d \sigma_{y}-\int_{\Omega} f \cdot \Phi d x .
\end{aligned}
$$

A density argument allows to conclude for every test fields in $H_{\Gamma}^{1}(\Omega)^{3} \times L^{2}\left(\Omega ; \mathcal{K}_{p e r}^{2}\left(\mathbf{Y}^{*}\right)\right)$.

Below, we prove the uniqueness of the solution of the unfolded problem (9.18).

Proposition 9.8. The problem (9.18) admits a unique solution $!^{4}$

\footnotetext{
${ }^{4}$ Proceeding as in Section 7 with first a constant non-negative friction $\widehat{G}$ belonging to $L^{2}(\Omega \times S)$ and then again using the Banach fixed-point theorem, one can prove the existence and the uniqueness of the solution to problem 9.18.
} 
Proof. Denote

$$
\mathbf{N}(v, \widehat{v})=\sqrt{\left(\left\|e(v)+e_{y}(\widehat{v})\right\|_{L^{2}\left(\Omega \times \mathbf{Y}^{*}\right)}^{2}+\left\|\nabla_{y} e_{y}(\widehat{v})\right\|_{L^{2}\left(\Omega \times \mathbf{Y}^{*}\right)}^{2}\right.}, \quad \forall(v, \widehat{v}) \in H_{\Gamma}^{1}(\Omega) \times L^{2}\left(\Omega ; H_{p e r}^{2}\left(\mathbf{Y}^{*}\right)^{3} \cap W\left(\mathbf{Y}^{*}\right)\right) .
$$

Due to Lemma 9.3, $\mathbf{N}$ is a norm over $H_{\Gamma}^{1}(\Omega) \times L^{2}\left(\Omega ; H_{p e r}^{2}\left(\mathbf{Y}^{*}\right)^{3} \cap W\left(\mathbf{Y}^{*}\right)\right)$.

First, note that the Theorem 9.7 gives a pair $(u, \widehat{u})$ which satisfies the problem (9.18).

Below we only detail the proof of the uniqueness.

Let $\left(u^{\prime}, \widehat{u}^{\prime}\right)$ be another solution of this problem. First, choose as test fields $(\Phi, \widehat{\phi})=\left(u^{\prime}, \widehat{u}^{\prime}\right)$ in $(9.18)$, then since $\left(u^{\prime}, \widehat{u}^{\prime}\right)$ is also a solution, in the corresponding problem chose as test fields $(\Phi, \widehat{\phi})=(u, \widehat{u})$. Finally, add both inequalities. That gives

$$
\begin{aligned}
& \int_{\Omega \times \mathbf{Y}^{*}} a_{i j k l}\left(e_{i j}\left(u-u^{\prime}\right)+e_{y, i j}\left(\widehat{u}-\widehat{u}^{\prime}\right)\right)\left(e_{k l}\left(u-u^{\prime}\right)+e_{y, k l}\left(\widehat{u}-\widehat{u}^{\prime}\right)\right) d x d y \\
+ & \kappa \int_{\Omega^{\prime} \times \mathbf{Y}^{*}}\left|\nabla_{y} e_{y}\left(\widehat{u}-\widehat{u}^{\prime}\right)\right|^{2} d x d y \leq \int_{\Omega \times S}-\mu\left(\sigma_{\nu}\left(u-u^{\prime}\right)+\sigma_{y, \nu}\left(\widehat{u}-\widehat{u}^{\prime}\right)\right)\left(\left|[\widehat{u}]_{\tau}\right|-\left|\left[\widehat{u}^{\prime}\right]_{\tau}\right|\right) d x d \sigma_{y} .
\end{aligned}
$$

Hence

$$
\begin{aligned}
\bar{\alpha}\left\|e\left(u-u^{\prime}\right)+e_{y}\left(\widehat{u}-\widehat{u}^{\prime}\right)\right\|_{L^{2}\left(\Omega \times \mathbf{Y}^{*}\right)}^{2}+\kappa\left\|\nabla_{y} e_{y}\left(\widehat{u}-\widehat{u}^{\prime}\right)\right\|_{L^{2}\left(\Omega \times \mathbf{Y}^{*}\right)}^{2} & \\
& \left.\leq\|\mu\|_{L^{\infty}(\Omega)} \| \sigma_{\nu}\left(u-u^{\prime}\right)+\sigma_{y, \nu}\left(\widehat{u}-\widehat{u}^{\prime}\right)\right)\left\|_{L^{2}(\Omega \times S)}\right\|[\widehat{u}]_{\tau}-\left[\widehat{u}^{\prime}\right]_{\tau} \|_{L^{2}(\Omega \times S)} .
\end{aligned}
$$

The above inequality, (3.8) and (6.4) (applied with the displacement $y \longrightarrow e\left(u-u^{\prime}\right)(x) y+\left(\widehat{u}-\widehat{u}^{\prime}\right)(x, y)$ defined for a.e. $x \in \Omega$ ) lead to

$$
\min \{\bar{\alpha}, \kappa\}\left(\mathbf{N}\left(u-u^{\prime}, \widehat{u}-\widehat{u}^{\prime}\right)\right)^{2} \leq\|\mu\|_{L^{\infty}(\Omega)} C_{1}\|\mathbf{a}\|_{W^{1, \infty}(S)} \mathbf{N}\left(u-u^{\prime}, \widehat{u}-\widehat{u}^{\prime}\right) C_{0}\left\|e_{y}\left(\widehat{u}-\widehat{u}^{\prime}\right)\right\|_{L^{2}\left(\Omega \times \mathbf{Y}^{*}\right)} .
$$

Condition 8.6 gives the uniqueness of the solution.

Due to the nonlinearity of the terms involving the tangential jumps, a homogenized problem can not be obtained.

\section{Annex}

In this section we denote $u_{\varepsilon, G, \kappa}$ the solution of 7.1 (resp. $U_{\varepsilon, G}$ the solution of (5.3)) with $G \in \mathcal{C}_{\varepsilon}^{* *}$.

Proposition 10.1. There exists $\left(u_{G}, \widehat{u}_{G}\right) \in H_{\Gamma}^{1}(\Omega)^{3} \times L^{2}\left(\Omega ; \mathcal{K}_{\text {per }}^{1}\left(\mathbf{Y}^{*}\right)\right)^{5}$ such that when $(\varepsilon, \kappa)$ goes to $(0,0)$ (resp. \& goes to 0)

$$
\begin{aligned}
& \mathcal{T}_{\varepsilon}^{*}\left(u_{\varepsilon, G, \kappa}\right) \longrightarrow u_{G} \quad \text { strongly in } L^{2}\left(\Omega ; H^{1}\left(\mathbf{Y}^{*}\right)\right)^{3}, \\
& \mathcal{T}_{\varepsilon}^{*}\left(\nabla u_{\varepsilon, G, \kappa}\right) \longrightarrow \nabla u_{G}+\nabla_{y} \widehat{u}_{G} \quad \text { strongly in } L^{2}\left(\Omega \times \mathbf{Y}^{*}\right)^{3 \times 3}, \\
\text { (resp. } & \mathcal{T}_{\varepsilon}^{*}\left(U_{\varepsilon, G}\right) \longrightarrow u_{G} \quad \text { strongly in } L^{2}\left(\Omega ; H^{1}\left(\mathbf{Y}^{*}\right)\right)^{3}, \\
& \left.\mathcal{T}_{\varepsilon}^{*}\left(\nabla U_{\varepsilon, G}\right) \longrightarrow \nabla u_{G}+\nabla_{y} \widehat{u}_{G} \quad \text { strongly in } L^{2}\left(\Omega \times \mathbf{Y}^{*}\right)^{3 \times 3} .\right)
\end{aligned}
$$

The couple $\left(u_{G}, \widehat{u}_{G}\right)$ is the unique solution of the following variational inequality:

$$
\begin{aligned}
& \int_{\Omega \times \mathbf{Y}^{*}} a_{i j k l}\left(e_{i j}\left(u_{G}\right)+e_{y, i j}\left(\widehat{u}_{G}\right)\right)\left(e_{k l}\left(\Phi-u_{G}\right)+e_{y, k l}\left(\widehat{\phi}-\widehat{u}_{G}\right)\right) d x d y \\
& \quad+\int_{\Omega \times S} G\left(\left|[\widehat{\phi}]_{\tau}\right|-\left|\left[\widehat{u}_{G}\right]_{\tau}\right|\right) d x d \sigma_{y} \geq \int_{\Omega} f \cdot\left(\Phi-u_{G}\right) d x, \\
& \forall(\Phi, \widehat{\phi}) \in H_{\Gamma}^{1}(\Omega)^{3} \times L^{2}\left(\Omega ; \mathcal{K}_{p e r}^{1}\left(\mathbf{Y}^{*}\right)\right) .
\end{aligned}
$$

\footnotetext{
${ }^{5}$ see 9.15 for the definition of this space
} 
Proof. First, recall that the estimates (9.1) hold. So, there exist a subsequence of $\{\varepsilon, \kappa\}$, still denoted $\{\varepsilon, \kappa\}$, and $\left(u_{G}, \widehat{u}_{G}\right) \in H_{\Gamma}^{1}(\Omega)^{3} \times L^{2}\left(\Omega ; \mathcal{K}_{p e r}^{1}\left(\mathbf{Y}^{*}\right)\right)$ such that

$$
\begin{aligned}
& \mathcal{T}_{\varepsilon}^{*}\left(u_{\varepsilon, G, \kappa}\right) \longrightarrow u_{G} \quad \text { strongly in } L^{2}\left(\Omega ; H^{1}\left(\mathbf{Y}^{*}\right)\right)^{3}, \\
& \mathcal{T}_{\varepsilon}^{*}\left(\nabla u_{\varepsilon, G, \kappa}\right) \rightarrow \nabla u_{G}+\nabla_{y} \widehat{u}_{G} \quad \text { weakly in } L^{2}\left(\Omega \times \mathbf{Y}^{*}\right)^{3 \times 3}, \\
& \sqrt{\kappa} \varepsilon \mathcal{T}_{\varepsilon}^{*}\left(\nabla e\left(u_{\varepsilon, G, \kappa}\right)\right) \rightarrow 0 \quad \text { weakly in } L^{2}\left(\Omega \times \mathbf{Y}^{*}\right)^{27}, \\
& \frac{1}{\varepsilon} \mathcal{T}_{\varepsilon}^{b}\left(\left[u_{\varepsilon, G, \kappa}\right]_{S_{\varepsilon}}\right) \rightarrow\left[\widehat{u}_{G}\right]_{S} \quad \text { weakly in } L^{2}\left(\Omega ; H^{1 / 2}(S)\right)^{3} .
\end{aligned}
$$

Due to the lower semi-continuity with respect to the weak topology and the above convergences for the unfolded sequences, we obtain

$$
\begin{gathered}
\liminf _{(\varepsilon, \kappa) \rightarrow(0,0)}\left(\mathbf{a}^{\varepsilon}\left(u_{\varepsilon, G, \kappa}, u_{\varepsilon, G, \kappa}\right)+\kappa \varepsilon^{2}\left\|\nabla e\left(u_{\varepsilon, G, \kappa}\right)\right\|_{L^{2}\left(\Omega_{\varepsilon}^{*}\right)}^{2}+\frac{1}{\varepsilon} \int_{\Omega \times S} \mathcal{T}_{\varepsilon}^{b}(G) \mathcal{T}_{\varepsilon}^{b}\left(\left|\left[u_{\varepsilon, G, \kappa}\right]_{\tau}\right|\right) d x d \sigma_{y}-\int_{\Omega_{\varepsilon}^{*}} f \cdot u_{\varepsilon, G, \kappa} d x\right) \\
\geq \int_{\Omega \times \mathbf{Y}^{*}} a_{i j k l}\left(e_{i j}\left(u_{G}\right)+e_{y, i j}\left(\widehat{u}_{G}\right)\right)\left(e_{k l}\left(u_{G}\right)+e_{y, k l}\left(\widehat{u}_{G}\right)\right) d x d y+\int_{\Omega \times S} G\left|\left[\widehat{u}_{G}\right]_{\tau}\right| d x d \sigma_{y}-\int_{\Omega} f \cdot u_{G} d x
\end{gathered}
$$

Now, consider the test displacement $v_{\varepsilon} \in \mathcal{K}_{\varepsilon}$ introduced in the proof of Theorem 9.7. One has

$$
\begin{aligned}
& \lim _{(\varepsilon, \kappa) \rightarrow(0,0)}\left(\mathbf{a}^{\varepsilon}\left(u_{\varepsilon, G, \kappa}, v_{\varepsilon}\right)+\kappa \varepsilon^{2}\left[\nabla e\left(u_{\varepsilon, G, \kappa}\right), \nabla e\left(v_{\varepsilon}\right)\right]_{\varepsilon}+\left(G,\left|\left[\left(v_{\varepsilon}\right)_{\tau_{\varepsilon}}\right]_{S_{\varepsilon}}\right|\right)_{\varepsilon}-\left(f, v_{\varepsilon}\right)\right) \\
= & \int_{\Omega \times \mathbf{Y}^{*}} a_{i j k l}\left(e_{i j}\left(u_{G}\right)+e_{y, i j}\left(\widehat{u}_{G}\right)\right)\left(e_{k l}(\Phi)+e_{y, k l}(\widehat{\phi})\right) d x d y+\int_{\Omega \times S} G\left|[\widehat{\phi}]_{\tau}\right| d x d \sigma_{y}-\int_{\Omega} f \cdot \Phi d x
\end{aligned}
$$

Finally, a density argument allows to obtain inequality (10.2). Since the problem (10.2) admits a unique solution, the whole sequences converge to their limits. As in [8], we prove that the convergences $(10.1)_{2,4}$ are strong convergences.

Proceeding in the same way with the sequence $\left\{U_{\varepsilon, G}\right\}_{\varepsilon}$, one obtains the same limit problem.

The homogenization of problem (5.2) (and also of problem (6.1) for small $\kappa$ ) remains an open problem.

\section{References}

[1] D. Cioranescu, A. Damlamian, and J. Orlik, Homogenization via unfolding in periodic elasticity with contact on closed and open cracks, Asymptot. Anal., 82 (2013), 201-232.

[2] P. Grisvard, Elliptic problems in nonsmooth domains. 1985

[3] C. Eck and J. Jarusek. Existence results for the semicoercive static contact problem with Coulomb friction. Nonlinear Anal., 42(6, Ser. A: Theory Methods):961-976, 2000.

[4] C. Eck, J. Jarusek, Krbec, Unilateral Contact Problems: Variational Methods and Existence Theorems, Springer, 2005.

[5] D. Cioranescu, A. Damlamian and G. Griso. The periodic unfolding method in homogenization, SIAM J. of Math. Anal., Vol. 40, 4 (2008), 1585-1620.

[6] D. Cioranescu, A. Damlamian and G. Griso. The Periodic Unfolding Method for partial differential problems.

[7] D. Cioranescu, A. Damlamian, P. Donato, G. Griso, and R. Zaki, The periodic unfolding method in domains with holes, SIAM J. of Math. Anal. Vol. 44, 2 (2012), 718-760.

[8] G. Griso, A. Migunova and J. Orlik, Homogenization via unfolding in periodic layer with contact, Asymptotic Analysis 99 (2016) 23-52.

[9] G. Griso, A. Migunova and J. Orlik, Asymptotic analysis for domains separated by a thin layer made of periodic vertical beams, Journal of Elasticity 128 (2), 291-331. 
[10] D. Cioranescu, A. Damlamian and J. Orlik, Homogenization via unfolding in periodic elasticity with contact on closed and open cracks, Asymptotic Analysis, Vol. 82, Issue 3-4, 2013

[11] G. Griso, J. Orlik, O. Sivak, Behavior of structures made of thick beams.

[12] A. Damlamian, Some unilateral Korn inequalities with application to a contact problem, C. R. Acad. Sci. Paris, Ser. I, Mathematical Problems in Mechanics, 2012

[13] J. Gobert, Une inégalité fondamentale de la théorie de l'élasticité, Bull. Soc. Roy. Sci. Liège, 31, 1962, $182-191$.

[14] O.A.Oleinik, A.S.Schamaev, G.A.Yosifian, Mathematical problems in elasticity and homogenization, NorthHolland Publishing Company - Amsterdam/London/New York/Tokyo, 1992.

[15] Gahn, M., Neuss-Radu, M., Knabner, P., Homogenization of Reaction-Diffusion Processes in a TwoComponent Porous Medium with Nonlinear Flux Conditions at the Interface. SIAM Journal of Applied Mathematics 76 (2016): 1819-1843. 